Review Article

\title{
Target Sestrin2 to Rescue the Damaged Organ: Mechanistic Insight into Its Function
}

\author{
Moein Ala $\mathbb{D}^{1}$ and Seyed Parsa Eftekhar ${ }^{2}$ \\ ${ }^{1}$ School of Medicine, Tehran University of Medical Sciences (TUMS), Tehran, Iran \\ ${ }^{2}$ Student Research Committee, Health Research Center, Babol University of Medical Sciences, Babol, Iran \\ Correspondence should be addressed to Moein Ala; moinala75@yahoo.com
}

Received 5 June 2021; Accepted 18 October 2021; Published 2 November 2021

Academic Editor: Mateusz Maciejczyk

Copyright (c) 2021 Moein Ala and Seyed Parsa Eftekhar. This is an open access article distributed under the Creative Commons Attribution License, which permits unrestricted use, distribution, and reproduction in any medium, provided the original work is properly cited.

\begin{abstract}
Sestrin2 is a stress-inducible metabolic regulator and a conserved antioxidant protein which has been implicated in the pathogenesis of several diseases. Sestrin 2 can protect against atherosclerosis, heart failure, hypertension, myocardial infarction, stroke, spinal cord injury neurodegeneration, nonalcoholic fatty liver disease (NAFLD), liver fibrosis, acute kidney injury (AKI), chronic kidney disease (CKD), and pulmonary inflammation. Oxidative stress and cellular damage signals can alter the expression of Sestrin2 to compensate for organ damage. Different stress signals such as those mediated by P53, Nrf2/ARE, HIF- $1 \alpha$, NF- $\kappa$ B, JNK/c-Jun, and TGF- $\beta /$ Smad signaling pathways can induce Sestrin 2 expression. Subsequently, Sestrin2 activates Nrf2 and AMPK. Furthermore, Sestrin2 is a major negative regulator of mTORC1. Sestrin2 indirectly regulates the expression of several genes and reprograms intracellular signaling pathways to attenuate oxidative stress and modulate a large number of cellular events such as protein synthesis, cell energy homeostasis, mitochondrial biogenesis, autophagy, mitophagy, endoplasmic reticulum (ER) stress, apoptosis, fibrogenesis, and lipogenesis. Sestrin2 vigorously enhances M2 macrophage polarization, attenuates inflammation, and prevents cell death. These alterations in molecular and cellular levels improve the clinical presentation of several diseases. This review will shed light on the beneficial effects of Sestrin2 on several diseases with an emphasis on underlying pathophysiological effects.
\end{abstract}

\section{Introduction}

Sestrin2, encoded by SESN2 gene, is a stress-inducible metabolic regulator and a conserved antioxidant protein that protects cells against reactive oxygen species (ROS). In response to oxidative stress, P53 induces the expression of Sestrin2 to prevent further cellular damage $[1,2]$. Furthermore, P53, in a Sestrin2-dependent manner, regulates major signaling pathways such as AMP-activated protein kinase (AMPK)/mammalian target of rapamycin complex 1 (mTORC1) and toll-like receptor (TLR) signaling pathways that are heavily involved in the regulation of several physiologic and pathologic processes such as metabolism, inflammation, and growth [2, 3]. However, recent studies have shown that Sestrin2 can function through P53-independent mechanisms [4]. Besides, it has been elucidated that nuclear factor erythroid 2-related factor 2 (Nrf2), a major transcription factor for several antioxidant genes, binds to antioxidant-response element (ARE) within SESN2 gene, thereby promoting the expression of Sestrin 2 and exerting cytoprotective effects against oxidative stress [5].

Sestrin2 inhibits nicotinamide adenine dinucleotide phosphate (NADPH) oxidase to decrease oxidative stress [3]. Furthermore, it was reported that Sestrin2 can modulate the expression of uncoupling protein 1 (Ucp1) through attenuation of oxidative stress [6]. Ucp1 regulates heat generation and energy expenditure; hence, Sestrin 2 can mechanistically modulate metabolic diseases [6].

The expression of Sestrin2 significantly increases during inflammatory response to protect against oxidative stress and confine the progressive organ damage [7, 8]. Consistently, Sestrin2 knockout is associated with a burst of oxidative stress, more severe inflammatory response, and exacerbation of disease manifestations $[7,8]$. Redistribution 
and increased expression of Sestrin2 act as endogenous defense mechanisms against organ damage [9]. Sestrin2 is also a major player for immune regulation [10]. In addition, it was shown that Sestrin2 is involved in the protective effects of numerous drugs [11-13]. The expression of Sestrin 2 decreases in older age, and its expression is negatively correlated with frailty and health status during senescence [14].

Accumulating evidence indicates that Sestrin2 plays a crucial role in the pathogenesis of several diseases, and pharmacological interventions to target it possess therapeutic value [15-17]. These findings are useful in determining the prognosis of several diseases and open new horizons for new therapeutic approaches. Understanding the biologic function of Sestrin2 and identifying its interactions with a wide variety of signaling pathways contribute to finding proper targets for drug development. Furthermore, comprehensive insight into the function of Sestrin2 in different organs is necessary for proper pharmacological intervention. Herein, this review discusses the beneficial effects of Sestrin 2 on several diseases such as atherosclerosis, heart failure, myocardial infarction, liver fibrosis, kidney diseases, neurodegeneration, and other diseases where evidence is abundant.

\section{Sestrin2 in Cardiovascular Diseases}

2.1. Atherosclerosis. Atherosclerosis is an age-related disease, and age is an independent risk factor for its occurrence [18]. Dyslipidemia, endothelial dysfunction, immune dysregulation, and chronic inflammation of vessels' wall have been implicated in the development and progression of atherosclerosis [19-22]. Oxidation of cellular proteins, DNA, and particularly lipids is a great contributor for atherosclerosis, and therapeutic approaches to alleviate oxidative stress have been suggested for the proper management of atherosclerosis [23]. Recent studies demonstrated that Sestrin2 can profoundly benefit cardiovascular health by preventing atherosclerosis, and several mechanisms have been proposed in this regard. Here, we explain how Sestrin2 increases during atherosclerosis to compensate and reverse the pathologic alterations [24].

Patients with carotid atherosclerotic plaques have significantly higher plasma levels of Sestrin 2 compared with subjects without carotid plaques. Further, plasma Sestrin2 positively and independently correlates with the presence and severity of carotid plaques [25]. Similarly, Kishimoto et al. revealed that patients with coronary artery disease have markedly higher plasma levels of Sestrin 2 compared with those without coronary artery disease. In addition, Sestrin2 level was significantly associated with the number of $>50 \%$ stenotic segments and severity score [26]. Sestrin2 level of $>16.0 \mathrm{ng} / \mathrm{mL}$ was significantly (95\% confidence of interval (CI), odds ratio (OR) 1.79 (1.09-2.95)) associated with atherosclerosis [26].

Pigment epithelium-derived factor (PEDF) could inhibit mTORC1 and induce autophagy in human umbilical vein endothelial cells by activating the P53/Sestrin2 signaling pathway. Silencing P53 or Sestrin2 abrogated the effect of
PEDF on endothelial cell autophagy [27]. PEDF also suppressed the inflammatory response of macrophages and stabilized atherosclerotic plaques [28]. Sestrin2, via inhibition of mTORC1, can induce autophagy in macrophages, thereby enhancing their M2 polarization [29]. Autophagy plays a pivotal role in the stability of atherosclerotic plaques. Inhibition of autophagy particularly in macrophages leads to increased oxidative stress and apoptosis in lesional macrophages and stimulates plaque necrosis [30].

Hwang et al. revealed that Sestrin 2 knockdown leads to a burst of oxidative stress; uncontrolled release of several proinflammatory cytokines (such as interleukin 6 (IL6) and tumor necrosis factor $\alpha$ (TNF- $\alpha)$ ) and chemokines (such as monocyte chemoattractant protein 1 (MCP-1)); endoplasmic reticulum stress (ER stress); and apoptosis of endothelial cells in the lipopolysaccharide- (LPS-) mediated inflammation model [31]. In LPS-mediated inflammation, Sestrin2 knockdown resulted in downregulation of AMPK and led to overactivation of nuclear factor kappa $B(N F-\kappa B)$ and increased expression of cell adhesion molecules such as intercellular adhesion molecule 1 (ICAM1), vascular cell adhesion molecular 1 (VCAM1), and E-selectin in endothelial cells. Moreover, an AMPK agonist completely reversed the aforementioned effects of LPS on Sestrin2 knockdown endothelial cells [31]. Sestrin2, through an AMPKdependent manner, restores endothelial nitric oxide synthase (eNOS) expression and improves nitric oxide (NO) production [32]. Dysfunctional and decreased expression of eNOS is a major driver of atherosclerosis, and therapeutic interventions to enhance eNOS expression or inhibit its uncoupling are protective against atherosclerosis [33].

Sestrin2 plays an important role in monocyte activation through the AMPK/mTORC1 pathway [34]. Sestrin2 activates AMPK, then it activates tuberous sclerosis complex 2 (TSC2) to inhibit mTORC1 [35]. In addition, Sestrin2 binds to GATOR2 to release GATOR1. Subsequently, GATOR1 binds to and inactivates RagB, a small GTPase essential for mTORC1 activation. Hence, in the presence of Sestrin2, GATOR1 can inhibit mTORC1 [36]. Moreover, Saxton et al. revealed that Sestrin2 is a leucine-sensitive regulator of mTORC1. In the presence of enough leucine amino acid, Sestrin2 will be released from GATOR2. Subsequently, GATOR2 attenuates the inhibitory effect of GATOR1 on mTORC1 (Figure 1) [37]. mTORC1 inhibition by Sestrin2 changes the balance between M1 and M2 macrophages, in favor of the M2 subtype. Overexpression of Sestrin2 in high glucose and dyslipidemic conditions could decrease CD80/CD68 and M1 macrophage-related cytokines such as IL6 and TNF- $\alpha$ and increase the release of M2 macrophagerelated markers such as IL10 and CD163/CD68 [34]. Consistently, Sestrin2 overexpression hinders foam cell formation and prevents monocyte adhesion to endothelial cells [34]. Sestrin 2 also decreases the release of matrix metalloproteinases (MMPs) such as MMP2 and MMP9, thereby enhancing the stability of atherosclerotic plaques [38]. Uncontrolled monocyte activation and foam cell formation are crucial for initial and later stages of atherosclerosis [39]. They lead to the production of a large amount of inflammatory cytokines as a major driver of atherosclerosis 


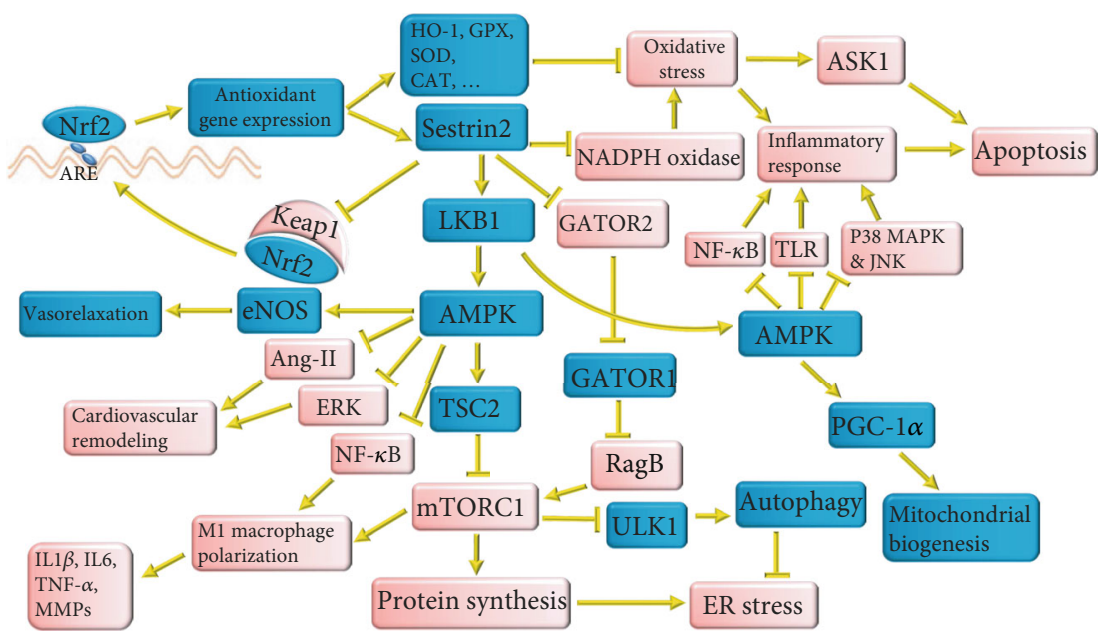

Figure 1: The molecular mechanisms underlying the protective effects of Sestrin2 on cardiovascular diseases. Sestrin 2 releases Nrf2 through autophagic degradation of Keap1. Activated Nrf2 binds to ARE of antioxidant genes and promotes the expression of Sestrin2 and other antioxidants. Sestrin 2 can inhibit NADHP oxidase. Attenuation of oxidative stress by Sestrin 2 and other antioxidants suppresses the inflammatory response and attenuates ASK1-mediated apoptosis. Sestrin2 also activates the LKB1/AMPK/TSC2 pathway to inhibit mTROC1. Besides, Sestrin2 attenuates the inhibitory effect of GATOR2 on GATOR1, thereby inhibiting mTORC1. mTORC1 activation accelerates protein synthesis leading to deposition of a large amount of misfolded protein during inflammation and stimulating ER stress. Furthermore, mTORC1 activation downregulates autophagy and mitophagy by inhibiting ULK1 and other activators of autophagy. Sestrin2-induced inhibition of mTORC1 enhances autophagy and mitophagy and inhibits ER stress. AMPK activation by Sestrin2 improves mitochondrial biogenesis through upregulation of PGC- $1 \alpha$. AMPK inhibits Ang-II- and ERK-induced cardiovascular remodeling. Sestrin 2 also attenuates oxidative stress and activates AMPK to inhibit TLR, NF- $\kappa$ B, JNK, and P38 MAPK and confine the inflammatory response.

in the initial stages. In the later stages, increased expression of MMPs by these macrophages leads to the rupture of atherosclerotic plaques [39]. It was uncovered that inhibition of mTORC1 can decrease macrophage infiltration, inflammatory cytokine release, and lesion size in atherosclerosis [40]. Therefore, the inhibitory effect of Sestrin2 on foam cells can strongly protect against atherosclerosis (Figure 2).

Metabolic syndrome is associated with a markedly increased risk of atherosclerosis [41]. It was shown that Sestrin2 positively correlates with body mass index (BMI), waist circumference, high-sensitivity C-reactive protein (hs-CRP), and body fat percentage. Besides, Sestrin2 was significantly higher in patients with type 2 diabetes and metabolic syndrome [42, 43]. It seems that metabolic syndrome and AMPK regulate Sestrin2 in a feedback mechanism [42]. Furthermore, as an activator of AMPK, metformin increases Sestrin2 expression [34, 44], and the AMPK antagonist, compound C, decreases Sestrin2 expression [34]. In return, Sestrin2 activates AMPK, improves insulin resistance, and alleviates metabolic syndrome [45]. Oxidized lipoprotein stimulates Sestrin2 expression through the c-Jun Nterminal kinase (JNK)/c-Jun pathway. Sestrin2 upregulation attenuates oxidized lipoprotein-mediated ROS production and apoptotic cell death (Figures 1 and 2) [46].

Excessive proliferation and apoptosis of vascular smooth muscle cells (VSMCs) are involved in the progression of atherosclerosis and the rupture of atherosclerotic plaques, respectively [47]. Sestrin2 inhibits mTORC1 in VSMCs to suppress their excessive proliferation. Furthermore, Sestrin2 inhibits ROS production and prevents excessive apoptosis of VSMCs [48]. Likewise, it was shown that Sestrin2 can upreg- ulate Nrf2 to protect VSMCs against Angiotensin-II- (AngII-) induced apoptosis (Figures 1 and 2) [49].

Endothelial progenitor cell (EPC) dysfunction is an important propellant of atherosclerosis. Ang-II induces apoptosis in EPCs and reduces Sestrin2 expression. Sestrin2 upregulation attenuates the deleterious effects of Ang-II on EPC and increases Nrf2 expression through P62-dependent autophagy [50]. In addition, Nrf2 silencing led to increased apoptosis of EPCs and EPC dysfunction through excessive ROS production [50]. Bae et al. uncovered that Sestrin2 can activate Nrf2, the chief regulator of antioxidant response, through autophagic degradation of Kelch-like ECH-associated protein 1 (Keap1) [51]. Releasing Nrf2 from Keap1 anchoring prevents its ubiquitination and subsequent proteolysis [51]. Nrf2, as a transcription factor, promotes the expression of several antioxidants such as Sestrin2 and heme oxygenase-1 (HO-1) by binding to their antioxidant response element (ARE) $[5,52,53]$. Nrf2 regulates physiological and pathophysiological processes in atherosclerosis, including lipid homeostasis, monocyte polarization, foam cell formation, and inflammation [54, 55]. Therefore, the mutual interaction between Nrf2 and Sestrin2 helps to maintain the protective function of Sestrin2 against oxidative stress (Figures 1 and 2).

2.2. Hypertension. Hypertension is a prevalent cardiovascular disease and consists of two subtypes, essential hypertension and secondary hypertension [56]. Essential hypertension is an age-related disease and constitutes $95 \%$ of hypertension cases [57]. Older age causes endothelial dysfunction due to oxidative stress and inflammation [58]. 

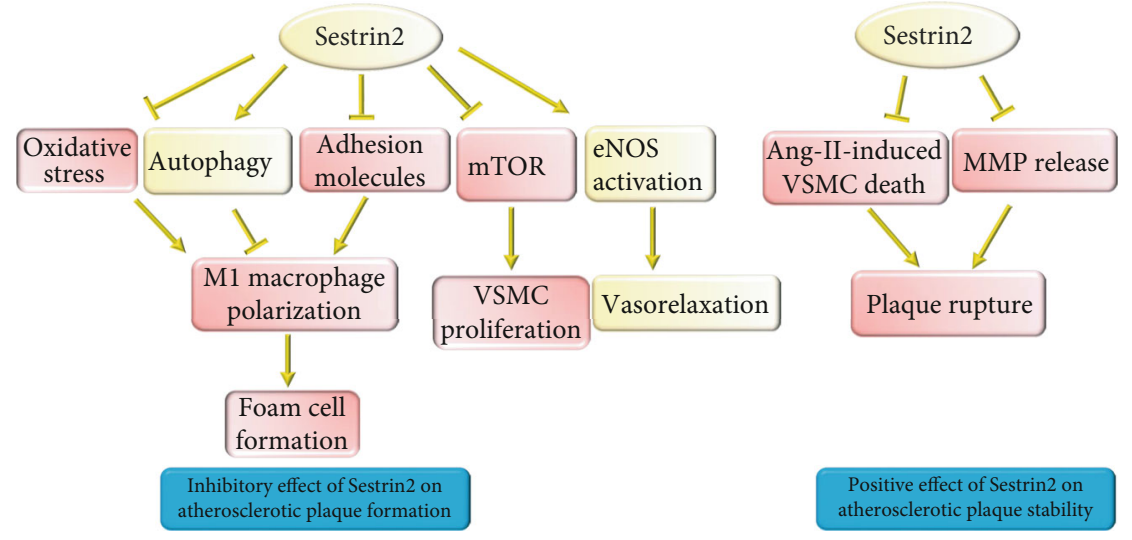

Figure 2: The protective effect of Sestrin2 on the formation and stability of atherosclerotic plaque. Sestrin2 attenuates oxidative stress and improves autophagy, thereby decreasing inflammation, downregulating the expression of adhesion molecules, and preventing M1 macrophage polarization and foam cell and plaque formation. Furthermore, Sestrin 2 can stabilize the preexisting plaques by preventing VSMC apoptosis and MMP release.

Additionally, increased renal ROS production plays a key role in the pathogenesis of hypertension [59]. Renal ROS activates the adrenergic system and the renin-angiotensinaldosterone system (RAAS) and leads to water-salt retention [59]. Sestrin2 attenuates renal ROS and contributes to blood pressure regulation. Yang et al. showed that silencing Sestrin2 with siRNA increases renal ROS production, hyperoxidized peroxiredoxins, lipid peroxidation, and both systolic blood pressure and diastolic blood pressure in mice [60]. Moreover, they revealed that dopamine receptor D2 (D2R) depends on Sestrin2 to attenuate oxidative stress or lower blood pressure [60].

Previously, it was mentioned that Sestrin2 can protect VSCMs against the deleterious effects of Ang-II [49]. Similarly, Sestrin2 can abrogate the detrimental effect of Ang-II on endothelial cells. Interestingly, Ang-II activates the JNK/c-Jun pathway to promote the expression of Sestrin2 (Figure 3). Subsequently, Sestrin2 attenuates Ang-IIinduced oxidative stress and apoptosis in endothelial cells. Consistently, silencing Sestrin2 could enhance the harmful effects of Ang-II on endothelial cells (Figure 3) [24]. Furthermore, Sestrin2, in an AMPK-dependent manner, inhibits Ang-II-induced phosphorylation of myosin phosphatase target subunit 1 (MYPT1) and myosin light chain (MLC) in VSMCs to lower blood pressure [61]. AMPK activation can vigorously abrogate Ang-II-induced hypertension [61]. Hence, Sestrin2/AMPK can strongly protect against Ang-II-mediated hypertension. Furthermore, Sestrin2 can inhibit mTORC1 which is heavily involved in vascular remodeling [62].

Endothelial dysfunction is a major contributor for hypertension, atherosclerosis, and other cardiovascular diseases. Endothelial dysfunction impairs endothelial-dependent vasorelaxation and provokes inflammatory response [63]. As mentioned previously, Sestrin2 can enhance eNOS function leading to vasorelaxation and modulation of endothelial dysfunction [32].

It was explained that Sestrin 2 can activate Nrf2, thereby enhancing the expression of itself and other antioxidants. Nrf2 is necessary for effective regulation of blood pressure,

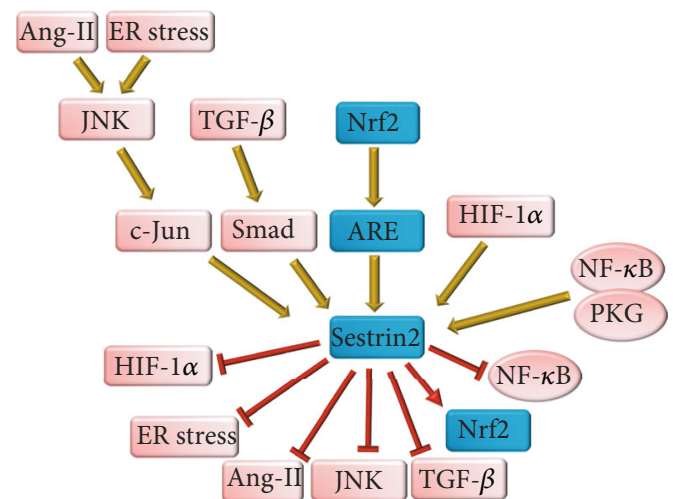

FIgURE 3: The autoregulatory effect of Sestrin2 on major signaling pathways. Several signaling pathways either protective such as Nrf2/ARE or destructive such as Ang-II, JNK/c-Jun, HIF- $1 \alpha$, NF$\kappa \mathrm{B}$, and TGF- $\beta /$ Smad can induce Sestrin 2 expression. In return, Sestrin2 selectively augments protective mechanisms and attenuates destructive signaling pathways.

and Nrf2 deletion or inhibition increases blood pressure in mice $[64,65]$. Nrf2 inhibition provokes oxidative stress, enhances inflammatory response, and exacerbates renal function [65]. Moreover, restoration of Nrf2 function alleviates renal inflammation, improves kidney function, and lowers blood pressure in rats $[65,66]$. Nrf2 prevents redox hypertensive effects by activating the antioxidant genes such as superoxide dismutase 1 (SOD1), catalase (CAT), glutathione peroxidase (GPX), and thioredoxin-1,2 (TRDX-1,2) (Figure 1) [67].

2.3. Arrhythmia. ROS are strongly involved in the pathophysiology of atrial fibrillation (AF) [68]. It was shown that patients with AF have higher plasma levels of malondialdehyde (MDA) and oxidized low-density lipoprotein (oxLDL) and lower levels of SOD, compared with healthy subjects [69]. Furthermore, these findings were more pronounced in patients with permanent $\mathrm{AF}$, compared with patients with paroxysmal and persistent AF. In addition, 
plasma levels of ROS were positively correlated with the size of the left atrium [69]. Oxidative stress leads to calcium overload, mitochondrial damage, RAAS system activation, and dysfunction of nitric oxide synthase [70]. Also, oxidative stress is the reason for the higher incidence of AF among patients with chronic inflammatory diseases such as diabetes [71]. Oxidative stress stimulates atrial fibroblast proliferation, and inhibition of oxidative stress can prevent atrial remodeling [71, 72]. Mitochondrial ROS can alter the phosphorylation levels of major ion transport proteins, thereby leading to electrical instability, ventricular arrhythmia, and sudden cardiac death [73]. Oxidative stress activates calcium-calmodulin-dependent protein kinase II (CaMKII) which subsequently leads to early afterdepolarization(EAD-) mediated AF [74]. CaMKII increases $\mathrm{Ca}^{2+}$ sparks and waves by phosphorylation of $\mathrm{Na}_{\mathrm{V}} 1.5$ in the atrium, leading to abnormal impulse generation [75]. $\mathrm{Na}_{\mathrm{V}} 1.5$ is a voltage-gated sodium channel subunit whose activation is associated with fast entry of $\mathrm{Na}^{+}$into cardiomyocytes and results in multifocal atrial and ventricular ectopy [76]. Consistently, it was shown that CaMKII is upregulated in the early stages of AF, and inhibition of CaMKII can prevent atrial hypertrophy and fibrosis [77].

However, Sestrin 2 cannot directly affect the heart's electrical function, but it can protect cardiomyocytes against oxidative stress and fibrosis [38, 78]. As mentioned, as a stimulator of cardiac remodeling, Ang-II changes the normal structure of atriums, particularly the left atrium, and induces AF [79]. Huang et al. showed that inhibition of Ang-II reduces the occurrence of AF in patients with hypertrophic cardiomyopathy [80]. Beyond its inhibitory effect on oxidative stress, Sestrin2 can suppress Ang-II-mediated alterations which protects against arrhythmias [24, 49].

2.4. Myocardial Ischemia-Reperfusion (IR). However, reperfusion is considered the best solution to decrease the destructive effects of ischemia, and it has its own destructive effects known as ischemia-reperfusion injury (IRI) [81]. Studies suggest that several mechanisms such as impaired mitochondrial biogenesis, mitochondrial calcium overload, mitochondrial fission, inadequate mitochondrial ATP production, uncontrolled ROS production, autophagy dysfunction, and platelet activation are involved in the pathogenesis of IRI [82-84]. Moreover, free radicals and cytokines produced by white blood cells aggravate IRI by damaging endothelial cells [85]. ROS are mainly produced in the reperfusion phase of myocardial IR. They can directly damage cell membrane and impair intracellular calcium homeostasis leading to $\mathrm{Ca}^{2+}$ overload and increased permeability of the mitochondrial membrane, known as mitochondrial permeability transition. These cellular events finally result in cardiomyocyte necrosis [86]. Similarly, ROS can activate major inflammatory molecules such as NF- $\kappa \mathrm{B}$ and trigger the release of several inflammatory cytokines such as IL1 $\beta$, IL6, and TNF- $\alpha$. These cytokines activate apoptotic cell death during IRI [86]. Moreover, ROS are involved in cardiac remodeling after myocardial infarction [86]. It was shown that Sestrin2, through attenuation of oxidative stress, can inhibit the activation of JNK and P38 mitogen-activated protein kinase (P38 MAPK) and subsequently downregulate $\mathrm{NF}-\kappa \mathrm{B}$ in myocardial IRI [87]. By attenuating NF- $\kappa \mathrm{B}$ signaling during myocardial IRI, Sestrin 2 vigorously decreased the uncontrolled release of inflammatory cytokines such as IL1 $\beta$, IL6, TNF- $\alpha$, INF- $\gamma$, and IL17. These inflammatory cytokines, especially IL17, can cause a reinforcement cycle of the inflammatory response inhibited by Sestrin2 (Figure 1) [87]. Interestingly, Sestrin2 also prevented $\mathrm{Ca}^{2+}$ induced mitochondrial permeability transition and restored membrane potential during myocardial IRI $[88,89]$. Sestrin2-mediated upregulation and activation of Nrf2 plays a pivotal role in attenuating oxidative stress in myocardial IRI $[88,89]$. Consistently, Nrf2 inhibition causes myocardial IRI to deteriorate [88].

Previous studies showed that Sestrin2 can confine the destructive effect of IRI, particularly myocardial IRI, by reprograming intracellular signaling pathways. Quan et al. showed that Sestrin2, in an LKB1-dependent manner, activates AMPK, thereby improving glucose uptake and oxidation and limiting the size of the infarct zone in the mice model of myocardial IR (Figure 1) [90]. AMPK alleviates myocardial IRI through the prevention of mitochondrial ROS production and inhibition of the c-Jun $\mathrm{N}$-terminal kinase (JNK) signaling pathway (Figure 1) [91]. In the absence of AMPK, overproduction of mitochondrial ROS and subsequent activation of JNK accelerate myocardial necrosis [91]. Morrison et al. observed that Sestrin2 expression increases in the early stages of myocardial ischemia and mitigates myocardial IRI through the LKB1/AMPK pathway. Sestrin2 knockout profoundly downregulated the LKB1/AMPK signaling pathway, increased infarct size, and impaired myocardial contractile function after IR [92]. Sestrin2 knockout is also associated with NF- $\kappa \mathrm{B}$ overactivity and increased expression of inflammatory cytokines such as IL1 $\beta$, IL17A, TNF- $\alpha$, and INF- $\gamma$ in the myocardium [87]. By inhibiting mTORC1, Sestrin2 attenuates M1 macrophage-mediated inflammatory response after myocardial infarction and enhances heart function [93]. Furthermore, Sestrin2-mediated inhibition of mTORC1 was shown to prevent IRI-induced ER stress during myocardial IRI. Indeed, inhibition of mTORC1 hinders the accumulation of unfolded proteins (Figure 1) [94]. Augmentation of inflammatory response due to Sestrin2 knockout impairs myocardial recovery after IRI and mimics age-related myocardial susceptibility to IRI [87].

Sestrin2 improves myocardial mitochondrial biogenesis after IRI. Herein, Sestrin 2 enhances the AMPK/PGC- $1 \alpha$ pathway and increases the expression of Ucp1 and mitochondrial transcription factor A (TFAM) [95, 96]. Ucp1 regulates mitochondrial ATP and ROS production as well as heat generation [97]. TFAM modulates calcium homeostasis and mitochondrial ROS production to confine myocardial injury after IRI. Increased expression of Ucp1 and TFAM can vigorously alleviate myocardial IRI [96, 98]. Interestingly, it was revealed that Sestrin2 is necessary for the competent function of oxidative phosphorylation of mitochondrial complexes under physiologic condition or I/R stress [89]. In the absence of Sestrin2, mitochondrial complexes and the Krebs cycle cannot effectively produce ATP 
[89]. Increased expression of Sestrin 2 during myocardial IRI is a compensatory mechanism to improve mitochondrial function and mitigate myocardial injury [89].

Mitophagy is a subtype of autophagy that helps to remove dysfunctional mitochondria and protect against myocardial IRI [99]. Kumar and Shaha showed that Sestrin2 stimulates unc-51-like kinase 1- (ULK1-) mediated phosphorylation of Beclin1 and potentiates the interaction between Beclin1 and Parkin. Subsequently, Parkin translocation on the mitochondrial surface activates mitophagy $[100,101]$.

2.5. Heart Failure and Cardiomyopathy. Oxidative stress has been introduced as the main driver of development and progression of heart failure. As mentioned previously, the increased amount of ROS impairs $\mathrm{Ca}^{2+}$ homeostasis and leads to contractile dysfunction [102]. ROS upregulate NF$\kappa \mathrm{B}$, P38 MAPK, and other signaling molecules to activate parallel inflammatory signals or activate apoptosis signalregulating kinase 1 (ASK1) that leads to apoptosis (Figure 1) [102]. Furthermore, ROS stimulate fibroblast proliferation and production of excessive amount of MMPs [102]. Cardiac fibroblasts secrete extracellular matrix (ECM) to maintain the structure and function of the myocardium under oxidative stress, pressure overload, and chronic ischemia. Cardiac fibroblasts play a crucial role in cardiac fibrosis and remodeling [103]. It was shown that the salivary levels of ROS increase with the progression of heart failure [104]. Patients with CHF have higher plasma levels of Sestrin2. Sestrin2 plasma levels also positively correlate with disease severity in patients with $\mathrm{CHF}$, and higher Sestrin2 levels predict increased incidence of major adverse cardiac events and poor outcome of the disease $[105,106]$. Similarly, it was shown that Sestrin2 increases in the rat model of doxorubicin-induced cardiomyopathy [106]. Besides, Sestrin2 knockout led to increased fibrogenesis and development of more severe pressure overload cardiac remodeling and hypertrophy in rats [107]. Sestrin 2 knockout disrupted mitochondrial function of cardiomyocytes and was associated with decreased glucose oxidation; decreased PGC- $1 \alpha$ and mitochondrial DNA; and elevated levels of 4-hydroxynonenal, a lipid peroxidation byproduct, in the rat model of pressure overload-mediated heart failure [107]. Sestrin 2 knockout decreased autophagy and increased apoptotic cell death of cardiomyocytes, as well [107].

Furthermore, Sestrin2 protects against cardiac hypertrophy by inhibiting mTORC1. mTORC1 phosphorylates and negatively regulates ULK1/2 and other autophagy-related molecules to inhibit autophagy (Figure 1) [107-110]. Agerelated reduction in Sestrin2 has been proposed as a probable mechanism for cardiac dysfunction and remodeling in older age [109]. A sufficient amount of autophagy is pivotal for the removal of dysfunctional organelles and nonfunctional proteins and the prevention of apoptosis in the heart [111]. Likewise, insufficient autophagy is associated with related cardiomyopathy [111].

Dong et al. revealed that Sestrin2 prevents cardiomyocyte hypertrophy through the downregulation of the extracellular signal-regulated kinase $1 / 2$ (ERK1/2) signaling pathway [112]. Prolonged activation of ERK causes mal- adaptive cardiac hypertrophy during pressure overload or anticancer chemotherapy (Figure 1) [113].

RAAS inhibitors can effectively prevent cardiac remodeling, decrease new-onset heart failure, reduce cardiovascular mortality and myocardial infarction, improve preexisting heart failure, and decrease cardiovascular death among patients with heart failure $[114,115]$. Herein, Sestrin2 can attenuate the deleterious effect of Ang-II on cardiomyocytes and prevent Ang-II-mediated apoptosis of cardiomyocytes (Figure 1) [116].

It was shown that Sestrin 2 and Sestrin2-mediated activation of $\mathrm{Nrf} 2$ can protect against pressure overload and obesity-induced cardiac remodeling in mice [11, 117]. Consistently, it was shown that $\mathrm{Nrf}^{-/-}$mice are more susceptible to cardiac remodeling, and Nrf 2 is protective against pressure overload-mediated, ischemia-induced, diabetes-mediated, and anticancer drug-mediated cardiac remodeling [118]. For instance, it was shown that Nrf2 knockout mice rapidly develop cardiac hypertrophy and heart failure following myocardial IRI or diabetes [119, 120].

Empagliflozin, a sodium-glucose cotransporter 2 (SGLT2) inhibitor, could mitigate obesity-induced cardiac dysfunction in mice [121]. It was shown that empagliflozin enhances the Sestrin2/AMPK/mTORC1 signaling pathway and augments the Nrf2/HO-1 pathway and eNOS activity to improve cardiac function [121]. In addition, the beneficial effects of empagliflozin were vigorously reversed in Sestrin2 knockout mice [121]. Interestingly, it was uncovered that SGLT-2 inhibitors can significantly decrease major adverse cardiovascular events, atherosclerotic cardiovascular disease, cardiovascular death and hospitalization because of heart failure, and progression of renal disease among diabetic patients [122].

Here, we explained how Sestrin2 is upregulated in several cardiovascular diseases to compensate for the damage. We also illustrated several mechanisms involved in the protective effects of Sestrin 2 on cardiovascular diseases. Regarding these findings, the clinical application of Sestrin2 in cardiovascular diseases is worth investigating.

\section{Sestrin2 in Liver Diseases}

3.1. NAFLD. Oxidative stress and lipid oxidation have been implicated in the pathogenesis of NAFLD, as the most common liver disease [123]. Patients with NAFLD and nonalcoholic steatohepatitis (NASH) have higher levels of oxidative stress, and treatment with antioxidants can partly alleviate liver steatosis and inflammation [123]. Previous studies revealed that Sestrin 2 protects against NAFLD and mediates the protective effects of different drugs on NAFLD. It was shown that carbon monoxide protects against hepatic steatosis by increasing Sestrin2 expression [124]. Sestrin2mediated inhibition of mTORC1 attenuated hepatic ER stress, hepatic steatosis, and fibrosis in mice fed with a high-fat diet $[125,126]$. Defective and insufficient autophagy response in patients with NASH or in mice fed with a highfat diet increases ER stress and leads to apoptotic cell death of hepatocytes [127, 128]. Pharmacological activation of autophagy and inhibition of ER stress prevented apoptosis 


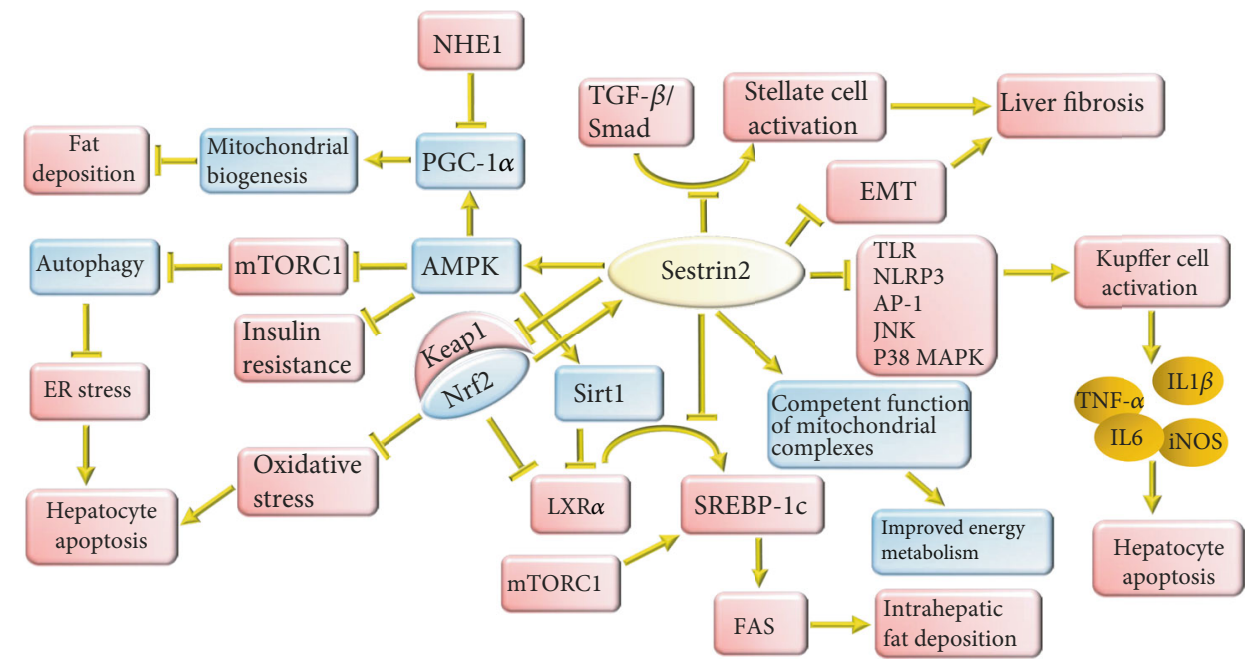

FIgURE 4: The protective effects of Sestrin2 on liver diseases. Similar to the cardiovascular system, Sestrin2 activates Nrf2 in the liver, thereby increasing the gene expression of itself and other antioxidants and attenuating oxidative stress. Also, Sestrin 2 can improve insulin resistance, autophagy, mitophagy, and ER stress by activating the AMPK/mTORC1 axis. Upregulation of PGC-1 $\alpha$ by Sestrin 2 helps to improve mitochondrial biogenesis and prevent NHE1-mediated intrahepatic fat accumulation. Furthermore, Sestrin2 can prevent de novo lipogenesis by attenuating LXR $\alpha$ - and mTORC1-mediated SREBP-1c upregulation. Activation of Nrf2 and APMK/Sirt1 signaling also contributes to inhibiting LXR $\alpha$-induced de novo lipogenesis. Sestrin2 is needed for competent function of mitochondrial complexes, and inadequate Sestrin2 expression impairs ATP production. Sestrin2 inhibits the major positive regulators of inflammatory signaling such as TLR, JNK, NLRP3, P38 MAPK, and AP-1 to prevent Kupffer cell activation and decrease cytokine release. Sestrin2 modulates TGF$\beta /$ Smad-mediated activation of stellate cells and hinders EMT to suppress liver fibrogenesis. These alterations alleviate liver inflammation, prevent intrahepatic fat deposition, and ameliorate liver fibrosis.

of hepatocytes and ameliorated NAFLD in mice fed with a high-fat diet (Figure 4) [129].

Previously, it was shown that glucagon-like peptide 1 (GLP1) agonists improved hepatic steatosis [130]. Liraglutide, a GLP1 agonist, increased the expression of Sestrin2 to protect against NAFLD. Sestrin2 activated the AMPK signaling pathway and augmented the Nrf2/HO- 1 axis to attenuate oxidative stress and suppress the inflammatory response [131]. In addition, Sestrin 2 knockout made hepatocytes susceptible to oxidative stress [51]. Sestrin2 binds to sequestosome-1 (SQSTM1) to accelerate autophagic degradation of Keap1 and activate Nrf2 [132]. SQSTM1 activates the AMPK/mTOR/ULK1 signaling pathway leading to autophagic degradation of Keap1 [132]. Nrf2 ${ }^{-/-}$decreases hepatic antioxidant expression, activates NF- $\kappa \mathrm{B}$, pronouncedly aggravates liver inflammation, and is associated with more severe hepatic steatosis [133]. Hence, Sestrin2, through the activation of $\mathrm{Nrf2}$, can partly increase the expression of itself and other antioxidants in the liver (Figure 4).

Increased expression of Sestrin 2 has been associated with the repression of liver X receptor $\alpha$ - (LXR $\alpha$-) mediated sterol regulatory element binding protein-1c (SREBP-1c) and fatty acid synthase (FAS) expression [12]. Liver X receptor- $\alpha$ $(\operatorname{LXR} \alpha)$ activates de novo synthesis of fatty acids in the liver. It upregulates SREBP-1c to increase the target gene expression for lipogenesis [12]. mTORC1 also upregulates SREBP-1c to enhance lipogenesis [134]. Additionally, Nrf2 and AMPK/NAD-dependent protein deacetylase sirtuin1 (SIRT1) signaling can inhibit LXR $\alpha$ and suppress $\operatorname{LXR} \alpha$ mediated de novo lipogenesis in the liver [135]. Hence, Ses- trin2 can mitigate NAFLD through direct or indirect downregulation of this major lipogenesis pathway (Figure 4).

Sestrin2 expression was shown to be negatively regulated by sodium-hydrogen exchanger 1 (NHE1). Indeed, a highfat diet increased NHE1 expression which was associated with hepatic steatosis. NHE1 knockout increased the gene expression of Sestrin 2 and PGC- $1 \alpha$ and ameliorated insulin resistance [136]. Furthermore, it was shown that Sestrin2 promoted the expression of PGC- $1 \alpha$ in liver cancer cells, and downregulation of Sestrin2 led to decreased expression of PGC- $1 \alpha$ [137]. Interestingly, it was demonstrated that PGC- $1 \alpha$ polymorphisms leading to its lower expression were contributory factors for the development of NAFLD [138]. It is probable that the improved function of Sestrin2 can attenuate the deleterious effects of NHE1 on PGC- $1 \alpha$ and hepatic steatosis (Figure 4). Meanwhile, Sestrin2, in an AMPKdependent manner, improves insulin resistance which finally results in decreased intrahepatic de novo lipogenesis and alleviates liver inflammation $[45,139]$.

3.2. Liver Fibrosis. In response to liver injury, liver macrophages known as Kupffer cells are the major source of ROS [140]. ROS produced by Kupffer cells induce necrosis and apoptosis of hepatocytes and stimulate hepatic stellate cells, leading to extracellular matrix deposition [140]. Therefore, inhibition of oxidative stress has been proposed as a therapeutic approach for liver fibrosis [140]. It was observed that patients with cirrhosis or mice with hepatic fibrosis have decreased expression of Sestrin2 in their liver [15, 141]. Sestrin2 inhibits hepatic stellate cell activation and prevents transforming growth factor $\beta$ - (TGF- $\beta$-) mediated liver 
fibrosis (Figure 4) [15, 142]. Sestrin2 overexpression has been associated with decreased expression of collagen $1 \mathrm{~A} 1$ and smooth muscle actin $\alpha$ (SMA- $\alpha)$ and reduced tissue level of inflammatory cytokines such as IL $1 \beta$ and TNF- $\alpha$ in the mice model of carbon tetrachloride- (CCl4-) induced liver fibrosis [142]. The TGF- $\beta$ signaling pathway promotes the expression of profibrotic genes such as collagen fibers, plasminogen activator inhibitor-1 (PAI-1), and connective tissue growth factor (CTGF) through Smad 2 and 3. Smads are transcription factors that can promote specific gene expression [143]. Interestingly, the upstream region of the SESN2 gene possesses a putative Smad-binding element (SBE) sequence, and it was shown that during overactivity of the TGF- $\beta$ signaling pathway, Smad increases Sestrin 2 expression. Sestrin 2 overexpression during the activation of the fibrogenic signaling of the TGF- $\beta /$ Smad axis could protect against liver fibrosis, but deletion of SBE decreased the expression of Sestrin 2 causing TGF- $\beta$-mediated liver fibrosis to deteriorate (Figure 3) [141]. Similarly, Sestrin2 overexpression was associated with reduced serum levels of alanine transaminase (ALT) and aspartate transaminase (AST) and prevented epithelial-mesenchymal transition (EMT) [142]. EMT expedites fibroblastic transformation of endothelial cells and progression of liver fibrosis [144]. Sestrin2 acts as a compensatory mechanism that is activated by the TGF$\beta /$ Smad signaling pathway and attempts to attenuate this signaling pathway. Exhaustion of Sestrin2 is usually found in the late stages of liver fibrosis [141].

Mice infected with the recombinant adenovirus SESN2 (Ad-SESN2) showed less severe hepatic injury in response to Gal/LPS-induced hepatic injury. Sestrin2 attenuated TLR-mediated inflammatory response and decreased the expression of inducible nitric oxide synthase (iNOS), IL1 $\beta$, IL6, and TNF- $\alpha$ [3]. Sestrin 2 profoundly ameliorates macrophage response by attenuating NLR family pyrin domain containing 3 (NLRP3) signaling and improving mitophagy (Figure 4) [145]. Sestrin2 could downregulate activator protein 1 (AP-1) [3]. AP-1 is a transcription factor whose activation by JNK and similar molecules promotes the expression of several inflammatory cytokines and leads to tissue damage (Figure 4) [146, 147].

The ameliorative effect of Sestrin2 on liver fibrosis was associated with the activation of AMPK signaling and downregulation of $\mathrm{mTORC1/S6/4E-BP}$ signaling [125, 142]. Previously, it was observed that the suppression of the mTORC1 signaling pathway is associated with apoptosis of activated hepatic stellate cells and protects against liver fibrosis [148]. It was shown that overexpression of Sestrin 2 can prevent tunicamycin-induced ER stress-mediated hepatocyte death. Indeed, Sestrin 2 negatively regulates $\mathrm{C} / \mathrm{EBP}$ homologous protein $(\mathrm{CHOP})$ to prevent ER stress-mediated apoptosis, and these effects were reversed in SESN2 ${ }^{+/-}$hepatocytes [125, 126]. In addition, as Sestrin 2 can inhibit mTORC1 signaling, it can prevent excessive production of proteins and decrease cell susceptibility to ER stress and unfolded protein response [125]. Consistently, it was observed that Sestrin2 knockdown aggravated ER stress, and inhibition of mTORC1 by rapamycin could alleviate ER stress [149]. Interestingly, it was shown that during ER stress, protein kinase regulated by the RNA-
(PKR-) like ER kinase (PERK)/CCAAT-enhancer-binding protein $\beta(\mathrm{c} / \mathrm{EBP} \beta)$ axis induces Sestrin 2 expression in hepatocytes (Figure 3) [125]. Kim et al. reported that during ER stress, the PERK/eukaryotic initiation factor- $2 \alpha$ (eIF2 $\alpha$ )/activating transcription factor 4 (ATF4) pathway can upregulate Sestrin2 expression in hepatocytes [124]. Jegal et al. also showed that activating transcription factor 6 (ATF6) can promote Sestrin2 expression to prevent ER stress-mediated apoptosis (Figure 3) [150]. Therefore, several members of ER stress can induce Sestrin 2 expression, while Sestrin 2 confines ER stress to prevent hepatocyte death.

In response to mitochondrial superoxide, Sestrin2, through ULK1, phosphorylates Beclin 1 at the serine-14 position, preparing it for binding to Parkin. Sestrin2 potentiates the interaction between Beclin1 and Parkin leading to the translocation of the cytosolic Parkin to the mitochondrial surface. The translocation of Parkin leads to the mitophagy of defective mitochondria [100]. Sestrin2-mediated autophagy and mitophagy can improve mitochondrial dysfunction and prevent hepatocyte apoptosis [151].

The liver needs Sestrin2 to modulate cellular homeostasis, oxidative stress, autophagy, mitophagy, and apoptosis and regulate a wide variety of cellular signaling pathways. Therapeutic interventions to enhance the expression of Sestrin 2 protects hepatocytes and can be a new candidate for the treatment of liver diseases.

\section{Sestrin2 in Kidney Diseases}

It was shown that Sestrin2 can protect against high-fat dietassociated renal fibrosis in rats. SESN2 deletion has been associated with podocyte loss and glomerular lesions in rats [152]. A significant increase in the serum levels of Sestrin2 was found in patients with diabetic nephropathy and metabolic syndrome [43]. Meanwhile, serum levels of Sestrin2 have been negatively associated with serum neutrophil gelatinase-associated lipocalin (NGAL) among healthy subjects and patients with type 2 diabetes with and without diabetic nephropathy [153]. Acute kidney injury, renal IR, and subsequent increase in oxidative stress activate the P53/Sestrin2 axis [154]. Sestrin2 plays a pivotal role in the attenuation of oxidative stress in acute kidney injury. Sestrin2 silencing augments oxidative stress and exacerbates kidney injury [60]. Sestrin 2 expression is necessary for an appropriate response to stress conditions in the kidney, and insufficient Sestrin 2 expression results in mTORC1 overactivity and, subsequently, apoptosis of renal cells. Downregulation of Sestrin2 in the kidney has been accompanied by glomerulosclerosis and severe periglomerular fibrosis in rats [155]. Sestrin2 overexpression alleviates renal I/R and increases autophagy and mitophagy in renal tubular cells [154]. Autophagy and mitophagy contribute to the removal of dysfunctional organelles and confine oxidative stress, ER stress, apoptosis, and necrosis in acute kidney injury and renal IRI [156]. Ineffective autophagy and mitophagy worsens tubular injury and renal function in acute kidney injury [156]. It was shown that Sestrin2 can attenuate ER stress in the kidney and prevent renal epithelial tubular cell 
EMT which is markedly involved in the progression of diabetic nephropathy [157].

Sestrin2 protects the kidney against oxidative stress by enhancing the Nrf2 function which simultaneously ameliorates hypertension [66]. In response to stress signals, Nrf2 upregulates the expression of several antioxidants such as glutathione, HO-1, glutathione S-transferase, and cytochromes to protect against AKI and CKD [158]. Sestrin2, a stress-inducible molecule, attempts to compensate for kidney injury and prevent renal fibrosis in patients with diabetic nephropathy [43].

\section{Sestrin2 in the Nervous System}

Higher oxygen consumption, higher abundance of polyunsaturated fatty acids, higher neuronal membrane surface area, using glutamate as a neurotransmitter, weak antioxidant defense, and several of other reasons make the nervous system more vulnerable to oxidative stress [159]. Microglial activation, mitochondrial dysfunction, and acceleration of oxidative stress are the leading causes of neuroinflammation that finally progresses to neurodegenerative diseases such as Alzheimer's disease, Parkinson's disease, and dementia [160].

5.1. Traumatic Brain Injury and Encephalitis. It was shown that the Sestrin2/Nrf2 pathway can improve traumatic brain injury by attenuating oxidative stress and apoptosis and decreasing brain edema and neurological deficit [161]. Luo et al. indicated that adenoassociated virus 2- (AAV2-) mediated Sestrin2 overexpression can improve sepsisassociated encephalitis in mice by enhancing AMPK $/ \mathrm{m}$ TORC1/ULK1-dependent autophagy [17]. Sestrin2 also decreased apoptosis of hippocampal neurons; lowered tissue levels of inflammatory cytokines such as IL1 $\beta$, IL6, and TNF- $\alpha$; and improved loss of learning and memory function in the mice model of sepsis-associated encephalopathy [17]. Previously, it was shown that autophagy can prevent microglial activation during sepsis-associated encephalitis and decrease inflammatory cytokine release [162].

5.2. Stroke and Hypoxic-Ischemic Encephalopathy. Sestrin2 promotes M2 macrophage polarization and inhibits M1 macrophage polarization after cerebral ischemia [29]. Contrary to M2 macrophage polarization, M1 macrophage polarization exacerbates oxygen glucose deprivationinduced neuronal death after ischemic stroke [163]. Sestrin2 could enhance AMPK/PGC- $1 \alpha$ to improve mitochondrial biogenesis in the rat model of cerebral I/R. Further, Sestrin2 downregulation aggravated oxidative stress, apoptosis, and neuronal damage and suppressed the AMPK/PGC- $1 \alpha$ and Nrf2 signaling pathway [164]. Moreover, Sestrin2 could block P38 MAPK-mediated expression of inflammatory cytokines such as IL1 $\beta$, IL6, IL18, and TNF- $\alpha$ in the cerebral I/R model [13].

Administration of human recombinant Sestrin2 improved neurologic function and restricted infarct size in the rat model of neonatal hypoxic-ischemic encephalopathy [165]. Sestrin 2 overexpression decreased the increased permeability of the blood-brain barrier and alleviated brain infarct and edema in the rat model of neonatal hypoxicischemic encephalopathy induced by common carotid artery ligation followed by 150 minutes of hypoxia. Besides, it was shown that hypoxia-inducible factor 1-alpha (HIF-1 $\alpha$ ) increased Sestrin2 expression in severe hypoxia-ischemia. Subsequently, Sestrin2 inhibited HIF- $1 \alpha$-mediated vascular endothelial growth factor (VEGF) expression and decreased blood-brain barrier permeability within the first 24 hours after hypoxic-ischemic injury (Figure 3) [166]. Early-onset increase in VEGF expression leads to the disruption of the blood-brain barrier following ischemic stroke and allows inflammatory cell infiltration, causes vasogenic edema, and exacerbates neuroinflammation, whereas late-onset increase in VEGF can improve the outcome of ischemic stroke [167, 168]. Interestingly, Sestrin2, through an Nrf2/HO-1dependent manner, could promote VEGF expression and angiogenesis in the rat model of ischemic stroke and decrease cerebral IRI, 5 and 10 days after ischemia [169, 170]. These findings show that Sestrin 2 may improve cerebral IRI by preventing early-onset increase in VEGF, while augmenting late-onset increase in VEGF.

5.3. Spinal Cord Injury and Peripheral Nerve Damage. Wu et al. uncovered that the protective effect of brain-derived neurotrophic factor (BDNF) on 3-nitropropionic acidinduced neuronal damage is associated with increased expression of Sestrin2 and attenuation of oxidative stress. Furthermore, it was shown that the production of NO, formation of cGMP, and subsequent activation of cGMPdependent protein kinase (PKG) are needed for BDNFmediated Sestrin 2 expression. The PKG, P50, and P65 subunits of NF- $\kappa \mathrm{B}$ form a complex that binds to the promoter of Sestrin 2 and upregulates its expression [171]. These interactions can justify how stress signals upregulate Sestrin2 and how Sestrin2 modulates them.

Spinal cord injury is associated with ER stress. PERK/ATF4 activation after spinal cord injury increases Sestrin2 expression. Sestrin2 via AMPK/mTORC1 enhances autophagy and prevents apoptosis and unfolded protein response. Sestrin2 overexpression ameliorates ER stress, improves neuronal survival, and promotes functional recovery after spinal cord injury. Additionally, AMPK inhibition weakens the protective effects of Sestrin2 on spinal cord injury [172]. It was shown that Sestrin2 expression increases in the mice model of neuropathic pain. In addition, Sestrin2 knockout was associated with significantly increased latephase neuropathic pain behavior and elevated ROS levels in the mice model of neuropathic pain. Furthermore, tertbutyl hydroperoxide, an ROS donor, stimulated prolonged pain behavior in naive $\mathrm{SESN}^{-/-}$mice [8].

5.4. Neurodegenerative Diseases like Alzheimer's Disease and Parkinson's Disease. The augmentation of AMPK has been proposed as a therapeutic mechanism for neurodegenerative diseases such as Parkinson's disease [173]. Meanwhile, increased oxidative stress has been vigorously implicated in the pathogenesis of neurodegenerative diseases [174]. Increased expression of Sestrin2 has been detected in the midbrain and serum of patients with Parkinson's disease 
[1, 175]. Additionally, it was shown that patients with Alzheimer's disease have higher serum protein and mRNA levels of Sestrin 2 compared with the normal control group and patients with mild cognitive impairment. Patients with mild cognitive impairment also had higher serum levels of Sestrin2 compared with the normal control group [176]. Consistently, increased expression of Sestrin2 was observed in the animal model of Parkinson's diseases induced by 1methyl-4-phenylpyridinium. Herein, 1-methyl-4-phenylpyridinium increased P53 expression, and subsequently, P53 provoked Sestrin2 expression [1]. SESN2 knockdown led to exacerbation of 1-methyl-4-phenylpyridinium-induced oxidative stress, mitochondrial dysfunction, and neuronal apoptosis [1]. Similarly, P53-mediated overexpression of Sestrin2 protected against sevoflurane-induced oxidative stress and apoptosis in neuronal cells [177]. Aging is accompanied by decreased expression of Sestrin2, and subsequently, a reduced capacity to compensate for neuronal damage and an increased risk of neurodegenerative diseases [178].

It was observed that amyloid $\beta$ peptide accumulation stimulates Sestrin2 expression in primary cortical neurons. Downregulation of Sestrin2 decreased amyloid $\beta$ peptidemediated autophagy in neuronal cells, increased amyloid $\beta$ peptide toxicity, and reduced cell survival [179]. It seems that Sestrin2 provides an endogenous protection against amyloid $\beta$ peptide neurotoxicity, and downregulation of Sestrin2 exacerbates neuronal damage after amyloid $\beta$ peptide exposure [179]. Sestrin 2 can enhance the longevity of neuronal cells and increase their tolerance to endogenous or exogenous damages.

5.5. Ototoxicity and Retinal Damage. It was observed that cochlear Sestrin2 expression decreases during older age. In addition, SESN2 knockout increased macrophage infiltration into the cochlea and accelerated age-related cochlear degeneration [178]. Ebnoether et al. revealed that gentamycininduced hair cell death is associated with downregulation of Sestrin2. Besides, Sestrin2 knockout was associated with greater hair cell loss after exposure to gentamycin [180]. The protective effect of gossypol acetic acid on the retinal pigmented layer was shown to be mediated through Sestrin2. Gossypol acetic acid led to Forkhead box O3 (FoxO3) nuclear translocation. Thereafter, FoxO3 promoted SESN2 transcription by binding to its enhancer [181]. Sestrin 2 overexpression also protected against hydrogen peroxideinduced retinal ganglion cell damage by upregulating Nrf2. Consistently, inhibition of Nrf2 abrogated the protective effects of Sestrin2 on retinal ganglion cells [182].

Accumulating evidence shows that Sestrin2 can protect against several neurologic diseases. It acts through various mechanisms to exert its protective effects. Lower expression of Sestrin2 during senescence can contribute to the development of neurodegenerative diseases which can be a new therapeutic target.

\section{Respiratory Diseases}

6.1. Obstructive Sleep Apnea (OSA). Higher rates of oxidative stress have been identified in patients with OSA. Moreover, oxidative stress positively correlates with the severity of OSA $[183,184]$. It was shown that patients with OSA have significantly higher plasma and urinary levels of Sestrin2. Plasma and urinary Sestrin 2 positively correlated with the apnea-hypopnea index (AHI) and the severity of OSA and negatively correlated with mean oxygen saturation. Furthermore, Sestrin2 levels significantly decreased after using continuous positive airway pressure (CPAP) for four weeks $[185,186]$. The plasma level of Sestrin2 has been proposed as a diagnostic and prognostic marker for OSA. Chai et al. proposed the Sestrin 2 plasma level of $1.86 \mathrm{ng} / \mathrm{mL}$ as a diagnostic cut-off value for OSA with a sensitivity of $81.58 \%$ and a specificity of $61.54 \%$. In addition, $5.21 \mathrm{ng} / \mathrm{mL}$ plasma Sestrin2 has been proposed as a cut-off value for severe OSA with a sensitivity of $61.90 \%$ and a specificity of $90.70 \%$ [187]. OSA is also complicated with several metabolic complications such as cardiovascular diseases in which Sestrin2 plays an ameliorative role [188]. Due to increased oxidative stress in patients with OSA, Sestrin2 has higher expression in these patients and protects them against cellular damage. Sestrin2 possesses a diagnostic and prognostic value and can be used for better management of OSA.

6.2. COPD and Asthma. Increased oxidative stress is a contributory factor for COPD and asthma which augments inflammation by activating NF- $\kappa \mathrm{B}$ and P38-MAPK, increases airway hyperresponsiveness, accelerates TGF- $\beta$ mediated fibrogenesis and airway remodeling, facilitates aging of the lungs and carcinogenesis, and enhances steroid resistance $[189,190]$.

It was reported that both serum level and tissue expression of Sestrin 2 are higher in patients with COPD. Furthermore, a positive correlation was found between serum Sestrin2 level and serum MMP9 level or airway remodeling in chest CT-scan [191]. Similarly, it was uncovered that patients with asthma have higher plasma levels of Sestrin2. A negative correlation was found between plasma levels of Sestrin 2 and $\mathrm{FEV}_{1} \%$ predicted and $\mathrm{FEV}_{1} / \mathrm{FVC}$ ratio during and after exacerbation of asthma [192]. In addition, higher levels of Sestrin2 have been identified in the sputum samples of patients with severe asthma, compared with patients with mild to moderate asthma [193].

It was observed that the anti-inflammatory effect of azithromycin on lung epithelial cells exposed to cigarette smoke extract is associated with upregulation of Sestrin2 [194]. Moreover, Sestrin2-mediated decrease in hyperoxidized peroxiredoxin could alleviate cigarette smokeinduced pulmonary alveolar type II epithelial cell injury [195]. In response to airway epithelial damage, HIF- $1 \alpha$ promotes Sestrin 2 expression, thereby confining epithelial barrier dysfunction [196].

Sestrin2 can lower inflammation and prevent fibrosis and airway remodeling in COPD. However, it was shown that attenuation of ROS, particularly superoxide anions through Sestrin2/Nrf2 signaling, can downregulate TGF$\beta /$ Smad and platelet-derived growth factor receptor-beta (PDGFR $\beta)$ and enhance cigarette smoke-mediated emphysema in the mice model [197-199]. Likewise, Sestrin2 


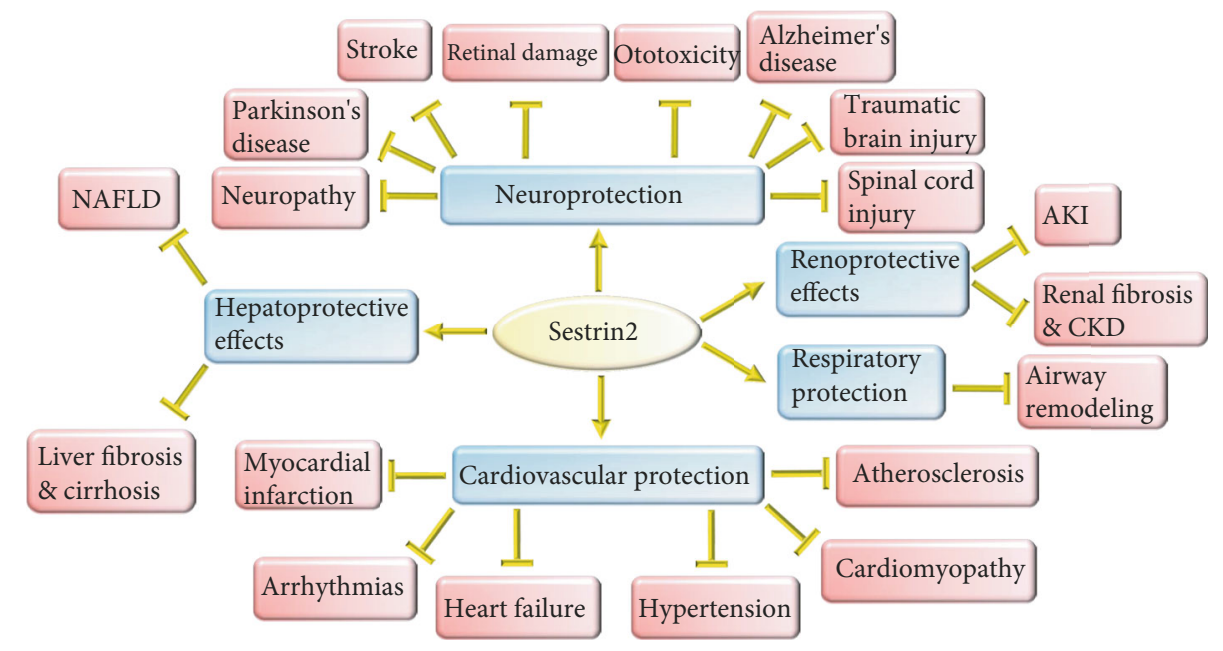

Figure 5: The protective effect of Sestrin2 on several organs. Sestrin 2 can alleviate the diseases of several organs. It enhances the function of cardiovascular system, nervous system, respiratory system, liver, and kidney. It alleviates inflammation, prevents airway remodeling in the respiratory system, protects against liver and renal fibrosis, and mitigates NAFLD and AKI. Furthermore, Sestrin 2 can profoundly improve cardiovascular and neuronal diseases. It ameliorates neuropathy, spinal cord injury, traumatic brain injury, retinal damage, hair cells damage, stroke, Parkinson's disease, and Alzheimer's disease. Sestrin2 can also enormously protect against myocardial infarction, arrhythmias, heart failure, atherosclerosis, hypertension, and cardiomyopathy.

inactivation could partly prevent pulmonary emphysema in these mice $[197,198]$.

Impaired autophagy response, either decreased or increased, has been implicated in the pathogenesis of COPD [200]. It was revealed that ROS-induced overexpression of Sestrin2 increases autophagy by activating AMPK/mTORC1/ULK1 in the mice exposed to cigarette smoke [201]. It was shown that AMPK deficiency exacerbates cigarette smoke-mediated lung inflammation and emphysema in mice $[202,203]$. In addition, Nrf2 activation could improve lung inflammation and emphysema, and Nrf2 deficiency was associated with more severe inflammation and emphysema in the mice model of cigarette smoke-induced lung inflammation $[203,204]$. The available evidence is inconsistent towards the effect of Sestrin2 and its downstream molecules on COPD, and future studies are needed to shed light on this issue.

\section{Sestrin2 Is an Autoregulatory Mechanism for Several Signaling Pathways}

In this review, we have mentioned several interactions between Sestrin 2 and other signaling pathways. It has been explained that some of these signaling pathways either protective or destructive can induce Sestrin2 expression. In return, Sestrin 2 showed that it can modulate these signaling pathways to provide the utmost benefit for the damaged tissue. Herein, Nrf2 can enhance Sestrin2 expression, and Sestrin2 can activate $\mathrm{Nrf} 2$ by releasing it from Keap1 sequestration. In addition, it was discussed that Ang-II or ER stress can induce Sestrin2 expression by several signaling pathways such as JNK signaling pathway. In return, Sestrin2 can inhibit ER stress and the JNK signaling pathway and attenuate Ang-II-mediated effects. The same scenario was found to happen for HIF- $1 \alpha$, TGF- $\beta$, and NF- $\kappa$ B. These findings show that Sestrin2 can provide an endogenous autoregulation mechanism for the proper function of the living cells. Sestrin 2 can be induced by inflammation and damage signals to attenuate them. Besides, it can strengthen the protective mechanisms against tissue damage (Figure 3).

\section{Can Sestrin2 Level Be Routinely Used for Clinical Purposes?}

Sestrin 2 can be routinely detected by serologic tests and available techniques such as the enzyme-linked immunosorbent assay (ELISA) method $[25,26]$. However, western blotting, polymerase chain reaction (PCR), and other molecular tests are applicable for measuring the expression of Sesrin2 in tissue biopsies particularly in cancers [205, 206]. Sestrin2 can accelerate the diagnosis of several diseases and help to estimate their prognosis $[25,26]$. For instance, it has been mentioned how Sestrin2 levels are associated with the presence or exacerbation of atherosclerosis, heart failure, myocardial infarction, diabetic nephropathy, and OSA in patients $[25,26,153,185]$. Ye et al. found that Sestrin2 plasma levels are positively correlated with the angiographic atherosclerosis score known as the Gensini score [207]. The Sestrin2 level can even differentiate between several differential diagnoses [208]. Interestingly, it was shown that the pleural effusion Sestrin2 level can successfully distinguish between malignant pleural effusion and congestive heart failure, tuberculosis, and parapneumonic pleural effusion [208]. Yet, studies could not show a strong correlation between the levels of Sestrin2 and disease-specific biomarkers such as troponin, creatinine, cystatin $\mathrm{C}$, and bilirubin. The functional role of Sestrin2 instead of being a structural protein and lack of tissue specificity can partly justify the absence of such correlation. 


\section{Conclusion and Future Direction}

Recent findings show that Sestrin2 is heavily involved in the pathogenesis of cardiovascular diseases, kidney diseases, liver diseases, respiratory diseases, and diseases of the nervous system and can improve the function of several organs (Figure 5). Primarily, Sestrin2 was considered just as an antioxidant; however, it was soon shown that Sestrin2 interacts with a wide variety of signaling pathways and regulates several cellular events such as autophagy, mitophagy, apoptosis, ER stress, mitochondrial biogenesis, lipogenesis, and fibrogenesis. Sestrin2 could enormously improve several diseases in vivo thanks to its vast molecular interactions. Pharmacological intervention to target Sestrin2 is rational in many chronic diseases and should be considered in future clinical trials.

\section{Data Availability}

Data sharing is not applicable for this narrative review. No data were generated and analyzed for this article.

\section{Conflicts of Interest}

The authors declare no conflicts of interest.

\section{Authors' Contributions}

Moein Ala conceptualized this article. Moein Ala and Seyed Parsa Eftekhar performed the literature search and wrote the draft. Moein Ala edited the article.

\section{References}

[1] Z. Daixing, Z. Chengye, Z. Qiang, and L. Shusheng, "Upregulation of sestrin-2 expression via P53 protects against 1methyl-4-phenylpyridinium (MPP+) neurotoxicity," Journal of Molecular Neuroscience, vol. 51, no. 3, pp. 967-975, 2013.

[2] W. Deng, J. Cha, J. Yuan et al., "p53 coordinates decidual sestrin 2/AMPK/mTORC1 signaling to govern parturition timing," The Journal of clinical investigation, vol. 126, no. 8, pp. 2941-2954, 2016.

[3] J. H. Yang, K. M. Kim, M. G. Kim et al., "Role of sestrin2 in the regulation of proinflammatory signaling in macrophages," Free Radical Biology and Medicine, vol. 78, pp. 156-167, 2015.

[4] G. T. Kim, S. H. Lee, J. I. Kim, and Y. M. Kim, "Quercetin regulates the sestrin 2-AMPK-p38 MAPK signaling pathway and induces apoptosis by increasing the generation of intracellular ROS in a p53-independent manner," International journal of molecular medicine, vol. 33, no. 4, pp. 863-869, 2014.

[5] B. Y. Shin, S. H. Jin, I. J. Cho, and S. H. Ki, "Nrf2-ARE pathway regulates induction of Sestrin-2 expression," Free Radical Biology and Medicine, vol. 53, no. 4, pp. 834-841, 2012.

[6] S.-H. Ro, M. Nam, I. Jang et al., "Sestrin2 inhibits uncoupling protein 1 expression through suppressing reactive oxygen species," Proceedings of the National Academy of Sciences, vol. 111, no. 21, pp. 7849-7854, 2014.

[7] Y. Liu, M. Li, M. Sun et al., "Sestrin2 is an endogenous antioxidant that improves contractile function in the heart during exposure to ischemia and reperfusion stress," Free Radical Biology and Medicine, vol. 165, pp. 385-394, 2021.

[8] W. Kallenborn-Gerhardt, R. Lu, K. M. J. Syhr et al., “Antioxidant activity of sestrin 2 controls neuropathic pain after peripheral nerve injury," Antioxidants \& redox signaling, vol. 19, no. 17, pp. 2013-2023, 2013.

[9] V. Soontornniyomkij, B. Soontornniyomkij, D. J. Moore et al., "Antioxidant sestrin-2 redistribution to neuronal soma in human immunodeficiency virus-associated neurocognitive disorders," Journal of Neuroimmune Pharmacology, vol. 7, no. 3, pp. 579-590, 2012.

[10] L.-X. Wang, X.-M. Zhu, and Y.-M. Yao, "Sestrin2: its potential role and regulatory mechanism in host immune response in diseases," Frontiers in immunology, vol. 10, 2019.

[11] J.-X. Du, J.-Z. Wu, Z. Li et al., "Pentamethylquercetin protects against cardiac remodeling via activation of Sestrin2," Biochemical and Biophysical Research Communications, vol. 512, no. 2, pp. 412-420, 2019.

[12] S. H. Jin, J. H. Yang, B. Y. Shin et al., "Resveratrol inhibits LXR $\alpha$-dependent hepatic lipogenesis through novel antioxidant _Sestrin2_ gene induction," Toxicology and applied pharmacology, vol. 271, no. 1, pp. 95-105, 2013.

[13] J. Liu, Y. Li, C. Mei et al., "Phytic acid exerts protective effects in cerebral ischemia-reperfusion injury by activating the antioxidative protein sestrin2," Bioscience, biotechnology, and biochemistry, vol. 84, no. 7, pp. 1401-1408, 2020.

[14] N. Rai, G. Venugopalan, R. Pradhan et al., "Exploration of novel anti-oxidant protein sestrin in frailty syndrome in elderly," Aging and disease, vol. 9, no. 2, pp. 220-227, 2018.

[15] J. H. Yang, K. M. Kim, S. S. Cho et al., "Inhibitory effect of Sestrin 2 on hepatic stellate cell activation and liver fibrosis," Antioxidants \& redox signaling, vol. 31, no. 3, pp. 243-259, 2019.

[16] H. S. Chae, M. Gil, S. K. Saha et al., "Sestrin2 expression has regulatory properties and prognostic value in lung cancer," Journal of personalized medicine, vol. 10, no. 3, p. 109, 2020.

[17] L. Luo, J. Wu, L. Qiao, G. Lu, J. Li, and D. Li, "Sestrin 2 attenuates sepsis-associated encephalopathy through the promotion of autophagy in hippocampal neurons," Journal of cellular and molecular medicine, vol. 24 , no. 12, pp. 66346643, 2020.

[18] J. C. Wang and M. Bennett, "Aging and atherosclerosis: mechanisms, functional consequences, and potential therapeutics for cellular senescence," Circulation Research, vol. 111, no. 2, pp. 245-259, 2012.

[19] J. W. Ding, "The effects of high mobility group box-1 protein on peripheral Treg/Th17 balance in patients with atherosclerosis," Acta Cardiol Sin, vol. 34, no. 5, pp. 399-408, 2018.

[20] Y. F. Sun, L. Li, Y. W. Wu, and K. P. Yang, "PD-1/PD-L1 in cardiovascular disease," Clinica Chimica Acta, vol. 505, pp. 26-30, 2020.

[21] H. S. Tsai, "The correlation between waist-hip ratio and achieving therapeutic lipid goals in Taiwan," Acta Cardiologica Sinica, vol. 35, no. 6, pp. 605-614, 2019.

[22] R. Menghini, R. Stöhr, and M. Federici, "MicroRNAs in vascular aging and atherosclerosis," Ageing Research Reviews, vol. 17, pp. 68-78, 2014.

[23] X. Yang, Y. Li, Y. Li et al., "Oxidative stress-mediated atherosclerosis: mechanisms and therapies," Frontiers in physiology, vol. $8,2017$. 
[24] L. Yi, F. Li, Y. Yong et al., "Upregulation of Sestrin-2 expression protects against endothelial toxicity of angiotensin II," Cell Biology and Toxicology, vol. 30, no. 3, pp. 147-156, 2014.

[25] Y. Kishimoto, E. Saita, R. Ohmori, K. Kondo, and Y. Momiyama, "Plasma sestrin2 concentrations and carotid atherosclerosis," Clinica Chimica Acta, vol. 504, pp. 56-59, 2020.

[26] Y. Kishimoto, M. Aoyama, E. Saita et al., "Association between plasma Sestrin2 levels and the presence and severity of coronary artery disease," Disease Markers, vol. 2020, 6 pages, 2020.

[27] T. Chen, T. Li, and J. Wang, "p53 mediates PEDF-induced autophagy in human umbilical vein endothelial cells through sestrin2 signaling," Molecular medicine reports, vol. 20, no. 2, pp. 1443-1450, 2019.

[28] H. Wen, M. Liu, Z. Liu et al., "PEDF improves atherosclerotic plaque stability by inhibiting macrophage inflammation response," International Journal of Cardiology, vol. 235, pp. 37-41, 2017.

[29] T. He, W. Li, Y. Song et al., "Sestrin2 regulates microglia polarization through mTOR-mediated autophagic flux to attenuate inflammation during experimental brain ischemia," Journal of neuroinflammation, vol. 17, no. 1, pp. 1-13, 2020.

[30] X. Liao, J. . C. Sluimer, Y. Wang et al., "Macrophage autophagy plays a protective role in advanced atherosclerosis," Cell metabolism, vol. 15, no. 4, pp. 545-553, 2012.

[31] H.-J. Hwang, T. W. Jung, J.-H. Choi et al., "Knockdown of sestrin2 increases pro-inflammatory reactions and ER stress in the endothelium via an AMPK dependent mechanism," Biochimica et Biophysica Acta (BBA)-Molecular Basis of Disease, vol. 1863, no. 6, pp. 1436-1444, 2017.

[32] A. A. Eid, D.-Y. Lee, L. J. Roman, K. Khazim, and Y. Gorin, "Sestrin 2 and AMPK connect hyperglycemia to Nox4dependent endothelial nitric oxide synthase uncoupling and matrix protein expression," Molecular and cellular biology, vol. 33, no. 17, pp. 3439-3460, 2013.

[33] F.-f. Hong, X.-y. Liang, W. Liu et al., "Roles of eNOS in atherosclerosis treatment," Inflammation Research, vol. 68, no. 6, pp. 429-441, 2019.

[34] S. Sundararajan, I. Jayachandran, M. Balasubramanyam, V. Mohan, B. Venkatesan, and N. Manickam, "Sestrin2 regulates monocyte activation through AMPK-mTOR nexus under high-glucose and dyslipidemic conditions," Journal of Cellular Biochemistry, vol. 120, no. 5, pp. 8201-8213, 2019.

[35] A. V. Budanov and M. Karin, "p53 target genes sestrin1 and sestrin2 connect genotoxic stress and mTOR signaling," Cell, vol. 134, no. 3, pp. 451-460, 2008.

[36] J. S. Kim, S.-H. Ro, M. Kim et al., "Sestrin2 inhibits mTORC1 through modulation of GATOR complexes," Scientific reports, vol. 5, no. 1, pp. 1-10, 2015.

[37] R. A. Saxton, K. E. Knockenhauer, R. L. Wolfson et al., "Structural basis for leucine sensing by the Sestrin2-mTORC1 pathway," Science, vol. 351, no. 6268, pp. 53-58, 2016.

[38] H.-J. Hwang, J. W. Kim, H. S. Chung et al., "Knockdown of Sestrin2 increases lipopolysaccharide-induced oxidative stress, apoptosis, and fibrotic reactions in $\mathrm{H} 9 \mathrm{c} 2$ cells and heart tissues of mice via an AMPK-dependent mechanism," Mediators Inflamm, vol. 2018, article 6209140, pp. 1-10, 2018.

[39] B. G. Childs, D. J. Baker, T. Wijshake, C. A. Conover, J. Campisi, and J. M. van Deursen, "Senescent intimal foam cells are deleterious at all stages of atherosclerosis," Science, vol. 354, no. 6311, pp. 472-477, 2016.

[40] D. Ai, H. Jiang, M. Westerterp et al., "Disruption of mammalian target of rapamycin complex 1 in macrophages decreases chemokine gene expression and atherosclerosis," Circulation Research, vol. 114, no. 10, pp. 1576-1584, 2014.

[41] M. J. Gurka, S. H. Golden, S. K. Musani et al., "Independent associations between a metabolic syndrome severity score and future diabetes by sex and race: the Atherosclerosis Risk In Communities Study and Jackson Heart Study," Diabetologia, vol. 60, no. 7, pp. 1261-1270, 2017.

[42] H. S. Chung, H.-J. Hwang, S. Y. Hwang et al., "Association of serum Sestrin2 level with metabolic risk factors in newly diagnosed drug-naive type 2 diabetes," Diabetes Res Clin Pract, vol. 144, pp. 34-41, 2018.

[43] H. M. el-Ashmawy and A. M. Ahmed, "Association of serum Sestrin-2 level with insulin resistance, metabolic syndrome, and diabetic nephropathy in patients with type 2 diabetes," The Egyptian Journal of Internal Medicine, vol. 31, no. 2, pp. 107-114, 2019.

[44] I. Ben-Sahra, B. Dirat, K. Laurent et al., "Sestrin2 integrates Akt and mTOR signaling to protect cells against energetic stress-induced death," Cell Death \& Differentiation, vol. 20, no. 4, pp. 611-619, 2013.

[45] H. Li, S. Liu, H. Yuan, Y. Niu, and L. Fu, "Sestrin 2 induces autophagy and attenuates insulin resistance by regulating AMPK signaling in $\mathrm{C} 2 \mathrm{C} 12$ myotubes," Experimental Cell Research, vol. 354, no. 1, pp. 18-24, 2017.

[46] H.-J. Hu, Z. Y. Shi, X. L. Lin, S. M. Chen, Q. Y. Wang, and S. Y. Tang, "Upregulation of Sestrin2 expression protects against macrophage apoptosis induced by oxidized lowdensity lipoprotein," DNA and Cell Biology, vol. 34, no. 4, pp. 296-302, 2015.

[47] M. R. Bennett, S. Sinha, and G. K. Owens, "Vascular smooth muscle cells in atherosclerosis," Circulation Research, vol. 118, no. 4, pp. 692-702, 2016.

[48] S. Lee, J. K. Byun, M. Park et al., "Melatonin inhibits vascular smooth muscle cell proliferation and apoptosis through upregulation of Sestrin2," Experimental and Therapeutic Medicine, vol. 19, no. 6, pp. 3454-3460, 2020.

[49] T. Xiao, L. Zhang, Y. Huang et al., "Sestrin2 increases in aortas and plasma from aortic dissection patients and alleviates angiotensin II-induced smooth muscle cell apoptosis via the Nrf2 pathway," Life Sciences, vol. 218, pp. 132-138, 2019.

[50] S. Ding, N. Ma, H. Liu, M. Tang, and J. Mei, “Sesn2 attenuates the damage of endothelial progenitor cells induced by angiotensin II through regulating the Keap1/Nrf2 signal pathway," Aging, vol. 12, no. 24, pp. 25505-25527, 2020.

[51] S. H. Bae, S. H. Sung, S. Y. Oh et al., "Sestrins activate Nrf2 by promoting p62-dependent autophagic degradation of Keap1 and prevent oxidative liver damage," Cell Metabolism, vol. 17, no. 1, pp. 73-84, 2013.

[52] K. Itoh, J. Mimura, and M. Yamamoto, "Discovery of the negative regulator of Nrf2, Keap1: a historical overview," Antioxidants \& Redox Signaling, vol. 13, no. 11, pp. 1665-1678, 2010.

[53] T. W. Kensler, N. Wakabayashi, and S. Biswal, "Cell survival responses to environmental stresses via the Keap1-Nrf2-ARE pathway," Annual Review of Pharmacology and Toxicology, vol. 47, no. 1, pp. 89-116, 2007. 
[54] T. E. Sussan, J. Jun, R. Thimmulappa et al., "Disruption of Nrf2, a key inducer of antioxidant defenses, attenuates ApoE-mediated atherosclerosis in mice," PloS one, vol. 3, no. 11, article e3791, 2008.

[55] J. Mimura and K. Itoh, "Role of Nrf2 in the pathogenesis of atherosclerosis," Free Radical Biology and Medicine, vol. 88, no. Part B, pp. 221-232, 2015.

[56] E. J. Benjamin, P. Muntner, A. Alonso et al., "Heart disease and stroke statistics-2019 update: a report from the American Heart Association," Circulation, vol. 139, no. 10, pp. e56e528, 2019.

[57] O. A. Carretero and S. Oparil, "Essential hypertension," Circulation, vol. 101, no. 3, pp. 329-335, 2000.

[58] T. W. Buford, "Hypertension and aging," Ageing Research Reviews, vol. 26, pp. 96-111, 2016.

[59] A. M. Briones and R. M. Touyz, "Oxidative stress and hypertension: current concepts," Current Hypertension Reports, vol. 12, no. 2, pp. 135-142, 2010.

[60] Y. Yang, S. Cuevas, S. Yang et al., "Sestrin2 decreases renal oxidative stress, lowers blood pressure, and mediates dopamine D2 receptor-induced inhibition of reactive oxygen species production," Hypertension, vol. 64, no. 4, pp. 825-832, 2014.

[61] X. Cao, T. Luo, X. Luo, and Z. Tang, "Resveratrol prevents AngII-induced hypertension via AMPK activation and RhoA/ROCK suppression in mice," Hypertension Research, vol. 37, no. 9, pp. 803-810, 2014.

[62] E. A. Goncharova, "mTOR and vascular remodeling in lung diseases: current challenges and therapeutic prospects," The FASEB Journal, vol. 27, no. 5, pp. 1796-1807, 2013.

[63] R. P. Brandes, "Endothelial dysfunction and hypertension," Hypertension, vol. 64, no. 5, pp. 924-928, 2014.

[64] L. Gao, M. C. Zimmerman, S. Biswal, and I. H. Zucker, "SelectiveNrf2Gene deletion in the rostral ventrolateral medulla evokes hypertension and sympathoexcitation in mice," Hypertension, vol. 69, no. 6, pp. 1198-1206, 2017.

[65] Z. Farooqui, R. S. Mohammad, M. F. Lokhandwala, and A. A. Banday, "Nrf2 inhibition induces oxidative stress, renal inflammation and hypertension in mice," Clinical and Experimental Hypertension, vol. 43, no. 2, pp. 175180, 2021.

[66] A. A. Javkhedkar, Y. Quiroz, B. Rodriguez-Iturbe, N. D. Vaziri, M. F. Lokhandwala, and A. A. Banday, "Resveratrol restored Nrf2 function, reduced renal inflammation, and mitigated hypertension in spontaneously hypertensive rats," American Journal of Physiology-Regulatory, Integrative and Comparative Physiology, vol. 308, no. 10, pp. R840-R846, 2015.

[67] J. H. Amaral, E. S. Rizzi, R. Alves-Lopes, L. C. Pinheiro, R. C. Tostes, and J. E. Tanus-Santos, "Antioxidant and antihypertensive responses to oral nitrite involves activation of the Nrf2 pathway," Free Radical Biology and Medicine, vol. 141, pp. 261-268, 2019.

[68] A. Samman Tahhan, P. B. Sandesara, S. S. Hayek et al., "Association between oxidative stress and atrial fibrillation," Heart Rhythm, vol. 14, no. 12, pp. 1849-1855, 2017.

[69] J. Y. Li, Y. He, H. H. Ke, Y. Jin, Z. Y. Jiang, and G. Q. Zhong, "Plasma oxidative stress and inflammatory biomarkers are associated with the sizes of the left atrium and pulmonary vein in atrial fibrillation patients," Clinical Cardiology, vol. 40, no. 2, pp. 89-94, 2017.
[70] X. Ren, X. Wang, M. Yuan et al., "Mechanisms and treatments of oxidative stress in atrial fibrillation," Current Pharmaceutical Design, vol. 24, no. 26, pp. 3062-3071, 2018.

[71] X. Liang, Q. Zhang, X. Wang et al., "Reactive oxygen species mediated oxidative stress links diabetes and atrial fibrillation," Molecular Medicine Reports, vol. 17, no. 4, pp. 49334940, 2018.

[72] Y. Yang, J. Zhao, J. Qiu et al., "Xanthine oxidase inhibitor allopurinol prevents oxidative stress-mediated atrial remodeling in alloxan-induced diabetes mellitus rabbits," Journal of the American Heart Association, vol. 7, no. 10, article e008807, 2018.

[73] S. Dey, D. DeMazumder, A. Sidor, D. B. Foster, and B. O'Rourke, "Mitochondrial ROS drive sudden cardiac death and chronic proteome remodeling in heart failure," Circulation Research, vol. 123, no. 3, pp. 356-371, 2018.

[74] P. A, C. H, F. MC, B. L, W. JN, and K. HS, “Atrial fibrillation initiated by early afterdepolarization-mediated triggered activity during acute oxidative stress: efficacy of late sodium current blockade," Journal of Heart Health, vol. 4, no. 1, 2018.

[75] A. Greer-Short, H. Musa, K. M. Alsina et al., "Calmodulin kinase II regulates atrial myocyte late sodium current, calcium handling, and atrial arrhythmia," Heart rhythm, vol. 17, no. 3, pp. 503-511, 2020.

[76] K. Calloe, A. K. Broendberg, A. H. Christensen et al., "Multifocal atrial and ventricular premature contractions with an increased risk of dilated cardiomyopathy caused by a $\mathrm{Na}_{\mathrm{v}} 1.5$ gain- of-function mutation (G213D)," International Journal of Cardiology, vol. 257, pp. 160-167, 2018.

[77] Z. Liu, J. E. Finet, J. A. Wolfram, M. E. Anderson, X. Ai, and J. K. Donahue, "Calcium/calmodulin-dependent protein kinase II causes atrial structural remodeling associated with atrial fibrillation and heart failure," Heart Rhythm, vol. 16, no. 7, pp. 1080-1088, 2019.

[78] Z. Dong, C. Lin, Y. Liu et al., "Upregulation of sestrins protect atriums against oxidative damage and fibrosis in human and experimental atrial fibrillation," Scientific Reports, vol. 7, no. 1, article 46307, 2017.

[79] H. J. Jansen, M. Mackasey, M. Moghtadaei et al., "Distinct patterns of atrial electrical and structural remodeling in angiotensin II mediated atrial fibrillation," Journal of Molecular and Cellular Cardiology, vol. 124, pp. 12-25, 2018.

[80] C.-Y. Huang, Y. H. Yang, L. Y. Lin et al., "Renin-angiotensinaldosterone blockade reduces atrial fibrillation in hypertrophic cardiomyopathy," Heart, vol. 104, no. 15, pp. 12761283, 2018.

[81] B. Ibáñez, G. Heusch, M. Ovize, and F. van de Werf, "Evolving therapies for myocardial ischemia/reperfusion injury," Journal of the American College of Cardiology, vol. 65, no. 14, pp. 1454-1471, 2015.

[82] E. Murphy and C. Steenbergen, "Mechanisms underlying acute protection from cardiac ischemia-reperfusion injury," Physiological Reviews, vol. 88, no. 2, pp. 581-609, 2008.

[83] D. J. Hausenloy and D. M. Yellon, "Myocardial ischemiareperfusion injury: a neglected therapeutic target," The Journal of Clinical Investigation, vol. 123, no. 1, pp. 92-100, 2013.

[84] H. Zhu, S. Toan, D. Mui, and H. Zhou, "Mitochondrial quality surveillance as a therapeutic target in myocardial infarction," Acta Physiologica, vol. 231, no. 3, article e13590, 2021. 
[85] R. A. Kloner, D. A. Brown, M. Csete et al., "New and revisited approaches to preserving the reperfused myocardium," Nature Reviews Cardiology, vol. 14, no. 11, pp. 679-693, 2017.

[86] M. Hori and K. Nishida, "Oxidative stress and left ventricular remodelling after myocardial infarction," Cardiovascular Research, vol. 81, no. 3, pp. 457-464, 2009.

[87] D. Ren, N. Quan, J. Fedorova, J. Zhang, Z. He, and J. Li, “Sestrin2 modulates cardiac inflammatory response through maintaining redox homeostasis during ischemia and reperfusion," Redox Biology, vol. 34, article 101556, 2020.

[88] X.-R. Zhou, X. C. Ru, C. Xiao et al., "Sestrin2 is involved in the Nrf2-regulated antioxidative signaling pathway in luteolin-induced prevention of the diabetic rat heart from ischemia/reperfusion injury," Food \& Function, vol. 12, no. 8, pp. 3562-3571, 2021.

[89] D. Ren, Z. He, J. Fedorova et al., "Sestrin2 maintains OXPHOS integrity to modulate cardiac substrate metabolism during ischemia and reperfusion," Redox Biology, vol. 38, article 101824, 2021.

[90] N. Quan, W. Sun, L. Wang et al., "Sestrin2 prevents agerelated intolerance to ischemia and reperfusion injury by modulating substrate metabolism," Faseb j, vol. 31, no. 9, pp. 4153-4167, 2017.

[91] V. G. Zaha, D. Qi, K. N. Su et al., “AMPK is critical for mitochondrial function during reperfusion after myocardial ischemia," Journal of Molecular and Cellular Cardiology, vol. 91, pp. 104-113, 2016.

[92] A. Morrison, L. Chen, J. Wang et al., "Sestrin2 promotes LKB1-mediated AMPK activation in the ischemic heart," The FASEB Journal, vol. 29, no. 2, pp. 408-417, 2015.

[93] K. Yang, C. Xu, Y. Zhang, S. He, and D. Li, "Sestrin2 suppresses classically activated macrophages-mediated inflammatory response in myocardial infarction through inhibition of mTORC1 signaling," Frontiers in Immunology, vol. 8, p. 728, 2017.

[94] X. Li, Z. Shen, and X. Y. Yu, "Letter by Li et al. Regarding Article, "Dectin-1 Contributes to myocardial Ischemia/Reperfusion Injury by Regulating Macrophage Polarization and Neutrophil Infiltration"," Circulation, vol. 140, no. 5, article A11473, 2019Suppl_1, 2019.

[95] N. Quan, L. Wang, X. Chen et al., "Sestrin2 prevents agerelated intolerance to post myocardial infarction via AMPK/PGC-1 $\alpha$ pathway," Journal of Molecular and Cellular Cardiology, vol. 115, pp. 170-178, 2018.

[96] G. H. Kunkel, P. Chaturvedi, and S. C. Tyagi, "Mitochondrial pathways to cardiac recovery: TFAM," Heart Failure Reviews, vol. 21, no. 5, pp. 499-517, 2016.

[97] Q. Chen, H. Zhang, Y. Cao et al., "Schisandrin B attenuates $\mathrm{CCl}<$ span style="font-size: $8.33333 \mathrm{px} ">4</$ span $>$-induced liver fibrosis in rats by regulation of Nrf2-ARE and TGF\&beta;/Smad signaling pathways," Drug Design, Development and Therapy, vol. Volume 11, p. 2179, 2017.

[98] J. Hoerter, M. D. M. Gonzalez-Barroso, E. Couplan et al., "Mitochondrial uncoupling protein 1 expressed in the heart of transgenic mice protects against ischemic-reperfusion damage," Circulation, vol. 110, no. 5, pp. 528-533, 2004.

[99] M. Yang, B. S. Linn, Y. Zhang, and J. Ren, "Mitophagy and mitochondrial integrity in cardiac ischemia-reperfusion injury," Biochimica et Biophysica Acta (BBA) - Molecular Basis of Disease, vol. 1865, no. 9, pp. 2293-2302, 2019.
[100] A. Kumar and C. Shaha, "SESN2 facilitates mitophagy by helping Parkin translocation through ULK1 mediated Beclin1 phosphorylation," Scientific Reports, vol. 8, no. 1, p. $615,2018$.

[101] P. Wang, L. Wang, J. Lu et al., "SESN2 protects against doxorubicin-induced cardiomyopathy via rescuing mitophagy and improving mitochondrial function," Journal of Molecular and Cellular Cardiology, vol. 133, pp. 125-137, 2019.

[102] E. Takimoto and D. A. Kass, "Role of oxidative stress in cardiac hypertrophy and remodeling," Hypertension, vol. 49, no. 2, pp. 241-248, 2007.

[103] T. Moore-Morris, N. Guimarães-Camboa, K. E. Yutzey, M. Pucéat, and S. M. Evans, "Cardiac fibroblasts: from development to heart failure," Journal of Molecular Medicine, vol. 93, no. 8, pp. 823-830, 2015.

[104] A. Klimiuk, A. Zalewska, R. Sawicki, M. Knapp, and M. Maciejczyk, "Salivary oxidative stress increases with the progression of chronic heart failure," Journal of Clinical Medicine, vol. 9, no. 3, p. 769, 2020.

[105] H. Wang, N. Li, X. Shao et al., "Increased plasma sestrin2 concentrations in patients with chronic heart failure and predicted the occurrence of major adverse cardiac events: a 36month follow-up cohort study," Clinica Chimica Acta, vol. 495, pp. 338-344, 2019.

[106] M. Wang, J. Liu, J. Qin et al., "Increased expression of Sestrin2 in human and experimental heart failure," International Journal of Clinical and Experimental Pathology, vol. 9, no. 8, pp. 8075-8082, 2016.

[107] N. Quan, X. Li, J. Zhang et al., "Substrate metabolism regulated by Sestrin2-mTORC1 alleviates pressure overloadinduced cardiac hypertrophy in aged heart," Redox Biology, vol. 36, article 101637, 2020.

[108] Y. Li, C. Chen, F. Yao et al., "AMPK inhibits cardiac hypertrophy by promoting autophagy via mTORC1," Archives of Biochemistry and Biophysics, vol. 558, pp. 79-86, 2014.

[109] N. Quan, T. Rousselle, X. Li, J. Liu, Q. Lu, and J. Li, “Abstract 10834: Sestrin2-mTORC1 Signaling Cascade Modulates AgeRelated Hypertrophy," Circulation, vol. 138, article A10834, Suppl_1, 2018.

[110] R. Li, Y. Huang, I. Semple, M. Kim, Z. Zhang, and J. H. Lee, "Cardioprotective roles of sestrin 1 and sestrin 2 against doxorubicin cardiotoxicity," American Journal of Physiology-Heart and Circulatory Physiology, vol. 317, no. 1, pp. H39-H48, 2019.

[111] G. R. Y. de Meyer, G. W. de Keulenaer, and W. Martinet, "Role of autophagy in heart failure associated with aging," Heart Failure Reviews, vol. 15, no. 5, pp. 423-430, 2010.

[112] B. Dong, R. Xue, Y. Sun, Y. Dong, and C. Liu, “Sestrin 2 attenuates neonatal rat cardiomyocyte hypertrophy induced by phenylephrine via inhibiting ERK1/2," Molecular and Cellular Biochemistry, vol. 433, no. 1-2, pp. 113-123, 2017.

[113] S. Gallo, A. Vitacolonna, A. Bonzano, P. Comoglio, and T. Crepaldi, "ERK: a key player in the pathophysiology of cardiac hypertrophy," International Journal of Molecular Sciences, vol. 20, no. 9, p. 2164, 2019.

[114] K. T. Weber, "Extracellular matrix remodeling in heart failure: a role for de novo angiotensin II generation," Circulation, vol. 96, no. 11, pp. 4065-4082, 1997.

[115] G. Savarese, P. Costanzo, J. G. F. Cleland et al., "A metaanalysis reporting effects of angiotensin-converting enzyme 
inhibitors and angiotensin receptor blockers in patients without heart failure," Journal of the American College of Cardiology, vol. 61, no. 2, pp. 131-142, 2013.

[116] X. Wang, C. Yang, X. Liu, and P. Yang, "The impact of microRNA-122 and its target gene Sestrin-2 on the protective effect of ghrelin in angiotensin II-induced cardiomyocyte apoptosis," RSC Advances, vol. 8, no. 18, pp. 10107-10114, 2018.

[117] J. du, W. He, C. Zhang et al., "Pentamethylquercetin attenuates cardiac remodeling via activation of the sestrins/Keap1/Nrf2 pathway in MSG-induced obese mice," BioMed Research International, vol. 2020, 10 pages, 2020.

[118] S. Zhou, W. Sun, Z. Zhang, and Y. Zheng, "The role of Nrf2mediated pathway in cardiac remodeling and heart failure," Oxidative Medicine and Cellular Longevity, vol. 2014, 16 pages, 2014.

[119] J. Strom and Q. M. Chen, "Loss of Nrf2 promotes rapid progression to heart failure following myocardial infarction," Toxicology and Applied Pharmacology, vol. 327, pp. 52-58, 2017.

[120] J. Gu, Y. Cheng, H. Wu et al., "Metallothionein is downstream of Nrf2 and partially mediates sulforaphane prevention of diabetic cardiomyopathy," Diabetes, vol. 66, no. 2, pp. 529-542, 2017.

[121] X. Sun, F. Han, Q. Lu et al., "Empagliflozin ameliorates obesity-related cardiac dysfunction by regulating Sestrin2mediated AMPK-mTOR signaling and redox homeostasis in high-fat diet-induced obese mice," Diabetes, vol. 69, no. 6, pp. 1292-1305, 2020.

[122] T. A. Zelniker, S. D. Wiviott, I. Raz et al., "SGLT2 inhibitors for primary and secondary prevention of cardiovascular and renal outcomes in type 2 diabetes: a systematic review and meta-analysis of cardiovascular outcome trials," The Lancet, vol. 393, no. 10166, pp. 31-39, 2019.

[123] Y. Sumida, E. Niki, Y. Naito, and T. Yoshikawa, "Involvement of free radicals and oxidative stress in NAFLD/NASH," Free Radical Research, vol. 47, no. 11, pp. 869-880, 2013.

[124] H. J. Kim, Y. Joe, S. K. Kim et al., "Carbon monoxide protects against hepatic steatosis in mice by inducing sestrin-2 via the PERK-eIF2 $\alpha$-ATF4 pathway," Free Radical Biology and Medicine, vol. 110, pp. 81-91, 2017.

[125] H. W. Park, H. Park, S. H. Ro et al., "Hepatoprotective role of Sestrin2 against chronic ER stress," Nature Communications, vol. 5, no. 1, p. 4233, 2014.

[126] K. H. Jegal, E. O. Kim, J. K. Kim et al., "Luteolin prevents liver from tunicamycin-induced endoplasmic reticulum stress via nuclear factor erythroid 2-related factor 2-dependent sestrin 2 induction," Toxicology and Applied Pharmacology, vol. 399, article 115036, 2020.

[127] Á. González-Rodríguez, R. Mayoral, N. Agra et al., "Impaired autophagic flux is associated with increased endoplasmic reticulum stress during the development of NAFLD," Cell Death \& Disease, vol. 5, no. 4, pp. e1179-e1179, 2014.

[128] Q. Zhang, Y. Li, T. Liang et al., "ER stress and autophagy dysfunction contribute to fatty liver in diabetic mice," International Journal of Biological Sciences, vol. 11, no. 5, pp. 559568, 2015.

[129] N. Nasiri-Ansari et al., "Empagliflozin attenuates nonalcoholic fatty liver disease (NAFLD) in high fat diet fed ApoE (-/-) mice by activating autophagy and reducing ER stress and apoptosis," International Journal of Molecular Sciences, vol. 22, no. 2, p. 818, 2021.
[130] D. J. Cuthbertson, A. Irwin, C. J. Gardner et al., "Improved glycaemia correlates with liver fat reduction in obese, type 2 diabetes, patients given glucagon-like peptide-1 (GLP-1) receptor agonists," PloS one, vol. 7, no. 12, article e50117, 2012.

[131] X. Han, C. Ding, G. Zhang et al., "Liraglutide ameliorates obesity-related nonalcoholic fatty liver disease by regulating Sestrin2-mediated Nrf2/HO-1 pathway," Biochemical and Biophysical Research Communications, vol. 525, no. 4, pp. 895-901, 2020.

[132] D. H. Lee, J. S. Park, Y. S. Lee et al., "SQSTM1/p62 activates NFE2L2/NRF2 via ULK1-mediated autophagic KEAP1 degradation and protects mouse liver from lipotoxicity," Autophagy, vol. 16, no. 11, pp. 1949-1973, 2020.

[133] S. Chowdhry, M. H. Nazmy, P. J. Meakin et al., "Loss of Nrf2 markedly exacerbates nonalcoholic steatohepatitis," Free Radical Biology and Medicine, vol. 48, no. 2, pp. 357-371, 2010.

[134] I. Bakan and M. Laplante, "Connecting mTORC1 signaling to SREBP-1 activation," Current Opinion in Lipidology, vol. 23, no. 3, pp. 226-234, 2012.

[135] H. Y. Kay, W. D. Kim, S. J. Hwang et al., "Nrf2 inhibits LXR $\alpha$ dependent hepatic lipogenesis by competing with FXR for acetylase binding," Antioxidants \& Redox Signaling, vol. 15, no. 8, pp. 2135-2146, 2011.

[136] V. Prasad, S. Chirra, R. Kohli, and G. E. Shull, "NHE1 deficiency in liver: implications for non-alcoholic fatty liver disease," Biochemical and Biophysical Research Communications, vol. 450, no. 2, pp. 1027-1031, 2014.

[137] A. Kumar, S. Giri, and C. Shaha, "Sestrin2 facilitates glutamine-dependent transcription of PGC- $1 \alpha$ and survival of liver cancer cells under glucose limitation," The FEBS Journal, vol. 285, no. 7, pp. 1326-1345, 2018.

[138] M. Yoneda, K. Hotta, Y. Nozaki et al., “Association between PPARGC1A polymorphisms and the occurrence of nonalcoholic fatty liver disease (NAFLD)," BMC Gastroenterology, vol. 8, no. 1, pp. 1-8, 2008.

[139] E. Bugianesi, S. Moscatiello, M. F. Ciaravella, and G. Marchesini, "Insulin resistance in nonalcoholic fatty liver disease," Current Pharmaceutical Design, vol. 16, no. 17, pp. 1941-1951, 2010.

[140] T. Luangmonkong, S. Suriguga, H. A. M. Mutsaers, G. M. M. Groothuis, P. Olinga, and M. Boersema, "Targeting oxidative stress for the treatment of liver fibrosis," Reviews of Physiology, Biochemistry and Pharmacology, vol. 175, pp. 71-102, 2018.

[141] J. H. Yang and S. H. Ki, "Sestrin2 inhibited the activation of hepatic stellate cells and liver fibrosis via blocking transforming growth factor- $\beta$ signaling," The FASEB Journal, vol. 31, article lb568, 2017.

[142] Y.-B. Hu, X. T. Ye, Q. Q. Zhou, and R. Q. Fu, “Sestrin 2 attenuates rat hepatic stellate cell (HSC) activation and liver fibrosis via an mTOR/AMPK-dependent mechanism," Cellular Physiology and Biochemistry, vol. 51, no. 5, pp. 2111-2122, 2018.

[143] K. L. Walton, K. E. Johnson, and C. A. Harrison, “Targeting TGF- $\beta$ mediated SMAD signaling for the prevention of fibrosis," Frontiers in Pharmacology, vol. 8, p. 461, 2017.

[144] K. Yu, Q. Li, G. Shi, and N. Li, "Involvement of epithelialmesenchymal transition in liver fibrosis," Saudi Journal of Gastroenterology: Official Journal of the Saudi Gastroenterology Association, vol. 24, no. 1, pp. 5-11, 2018. 
[145] M.-J. Kim, S. H. Bae, J. C. Ryu et al., "SESN2/sestrin2 suppresses sepsis by inducing mitophagy and inhibiting NLRP3 activation in macrophages," Autophagy, vol. 12, no. 8, pp. 1272-1291, 2016.

[146] D. Morse, S. E. Pischke, Z. Zhou et al., "Suppression of Inflammatory Cytokine Production by Carbon Monoxide Involves the JNK Pathway and AP-1*," Journal of Biological Chemistry, vol. 278, no. 39, pp. 36993-36998, 2003.

[147] H. B. Schonthaler, J. Guinea-Viniegra, and E. F. Wagner, "Targeting inflammation by modulating the Jun/AP-1 pathway," Annals of the Rheumatic Diseases, vol. 70, Suppl 1, pp. i109-i112, 2011.

[148] Y.-L. Wu, Y. J. Zhang, Y. L. Yao et al., "Cucurbitacin E ameliorates hepatic fibrosis in vivo and in vitro through activation of AMPK and blocking mTOR-dependent signaling pathway," Toxicology Letters, vol. 258, pp. 147-158, 2016.

[149] Y. Liu, S. Li, Z. Gao et al., "Indoleamine 2,3-dioxygenase 1 (IDO1) promotes cardiac hypertrophy via a PI3K-AKTmTOR-dependent mechanism," Cardiovascular Toxicology, vol. 21, no. 8, pp. 655-668, 2021.

[150] K. H. Jegal, S. M. Park, S. S. Cho et al., "Activating transcription factor 6-dependent sestrin 2 induction ameliorates ER stress-mediated liver injury," Biochimica et Biophysica Acta (BBA)-Molecular Cell Research, vol. 1864, no. 7, pp. 12951307, 2017.

[151] K. H. Jegal, H. L. Ko, S. M. Park et al., "Eupatilin induces Sestrin2-dependent autophagy to prevent oxidative stress," Apoptosis, vol. 21, no. 5, pp. 642-656, 2016.

[152] A. Agouni, D. Y. Lee, A. A. Eid, Y. Gorin, and K. Sharma, "The protective role of Sestrin2 in high fat diet-induced nephropathy," in University of the Future: Re-Imagining Research and Higher Education, Qatar, 2020.

[153] K. M. Mohany and O. Al Rugaie, "Association of serum sestrin 2 and betatrophin with serum neutrophil gelatinase associated lipocalin levels in type 2 diabetic patients with diabetic nephropathy," Journal of Diabetes \& Metabolic Disorders, vol. 19, no. 1, pp. 249-256, 2020.

[154] M. Ishihara, M. Urushido, K. Hamada et al., "Sestrin-2 and BNIP3 regulate autophagy and mitophagy in renal tubular cells in acute kidney injury," American Journal of Physiology-Renal Physiology, vol. 305, no. 4, pp. F495-F509, 2013.

[155] H. Hamatani, K. Hiromura, T. Sakairi et al., "Expression of a novel stress-inducible protein, sestrin 2, in rat glomerular parietal epithelial cells," American Journal of PhysiologyRenal Physiology, vol. 307, no. 6, pp. F708-F717, 2014.

[156] G. P. Kaushal and S. V. Shah, "Autophagy in acute kidney injury,” Kidney International, vol. 89, no. 4, pp. 779-791, 2016.

[157] Y. Jia, Z. Zheng, Y. Yang et al., "MiR-4756 promotes albumin-induced renal tubular epithelial cell epithelial-tomesenchymal transition and endoplasmic reticulum stress via targeting Sestrin2," Journal of Cellular Physiology, vol. 234, no. 3, pp. 2905-2915, 2019.

[158] H. Saito, "Toxico-pharmacological perspective of the Nrf2Keap1 defense system against oxidative stress in kidney diseases," Biochemical Pharmacology, vol. 85, no. 7, pp. 865872, 2013.

[159] J. Friedman, "Why is the nervous system vulnerable to oxidative stress?," in Oxidative Stress and Free Radical Damage in Neurology, pp. 19-27, Springer, 2011.
[160] A. Reynolds, C. Laurie, R. Lee Mosley, and H. E. Gendelman, "Oxidative stress and the pathogenesis of neurodegenerative disorders," International Review of Neurobiology, vol. 82, pp. 297-325, 2007.

[161] X. Liu, M. Li, J. Zhu, W. Huang, and J. Song, "Sestrin2 protects against traumatic brain injury by reinforcing the activation of Nrf2 signaling," Human \& Experimental Toxicology, vol. 40, no. 7, pp. 1095-1111, 2021.

[162] X. Zhuang, Y. Yu, Y. Jiang et al., "Molecular hydrogen attenuates sepsis-induced neuroinflammation through regulation of microglia polarization through an mTOR-autophagydependent pathway," International Immunopharmacology, vol. 81, p. 106287, 2020.

[163] X. Hu, P. Li, Y. Guo et al., “Microglia/macrophage polarization dynamics reveal novel mechanism of injury expansion after focal cerebral ischemia," Stroke, vol. 43, no. 11, pp. 3063-3070, 2012.

[164] L. Li, L. Xiao, Y. Hou et al., "Sestrin2 silencing exacerbates cerebral ischemia/reperfusion injury by decreasing mitochondrial biogenesis through the AMPK/PGC- $1 \alpha$ pathway in rats," Scientific Reports, vol. 6, no. 1, pp. 1-11, 2016.

[165] X. Shi, L. Xu, D. M. Doycheva, J. Tang, M. Yan, and J. H. Zhang, "Sestrin2, as a negative feedback regulator of mTOR, provides neuroprotection by activation AMPK phosphorylation in neonatal hypoxic-ischemic encephalopathy in rat pups," Journal of Cerebral Blood Flow \& Metabolism, vol. 37, no. 4, pp. 1447-1460, 2017.

[166] X. Shi, D. M. Doycheva, L. Xu, J. Tang, M. Yan, and J. H. Zhang, "Sestrin2 induced by hypoxia inducible factor1 alpha protects the blood-brain barrier via inhibiting VEGF after severe hypoxic-ischemic injury in neonatal rats," Neurobiology of Disease, vol. 95, pp. 111-121, 2016.

[167] C. Yang, K. E. Hawkins, S. Doré, and E. Candelario-Jalil, "Neuroinflammatory mechanisms of blood-brain barrier damage in ischemic stroke," American Journal of Physiology-Cell Physiology, vol. 316, no. 2, pp. C135-C153, 2019.

[168] J.-P. Yang, H.-J. Liu, and X.-F. Liu, "VEGF promotes angiogenesis and functional recovery in stroke rats," Journal of Investigative Surgery, vol. 23, no. 3, pp. 149-155, 2010.

[169] Y. Li, J. Wu, S. Yu et al., "Sestrin2 promotes angiogenesis to alleviate brain injury by activating Nrf2 through regulating the interaction between p62 and Keap1 following photothrombotic stroke in rats," Brain Research, vol. 1745, article 146948, 2020.

[170] P. Wang, Y. Zhao, Y. Li et al., "Sestrin2 overexpression attenuates focal cerebral ischemic injury in rat by increasing Nrf2/HO-1 pathway-mediated angiogenesis," Neuroscience, vol. 410, pp. 140-149, 2019.

[171] C.-L. Wu, S. D. Chen, J. H. Yin, C. S. Hwang, and D. I. Yang, "Nuclear factor-kappaB-dependent sestrin2 induction mediates the antioxidant effects of BDNF against mitochondrial inhibition in rat cortical neurons," Molecular Neurobiology, vol. 53, no. 6, pp. 4126-4142, 2016.

[172] Y. Li, J. Zhang, K. Zhou et al., "Elevating sestrin2 attenuates endoplasmic reticulum stress and improves functional recovery through autophagy activation after spinal cord injury," Cell Biology and Toxicology, vol. 37, no. 3, pp. 401-419, 2021.

[173] D. W. Curry, B. Stutz, Z. B. Andrews, and J. D. Elsworth, "Targeting AMPK signaling as a neuroprotective strategy in Parkinson's disease," Journal of Parkinson's Disease, vol. 8, no. 2, pp. 161-181, 2018. 
[174] A. Singh, R. Kukreti, L. Saso, and S. Kukreti, "Oxidative stress: a key modulator in neurodegenerative diseases," Molecules, vol. 24, no. 8, p. 1583, 2019.

[175] N. Rai, A. D. Upadhyay, V. Goyal, S. Dwivedi, A. B. Dey, and S. Dey, "Sestrin2 as serum protein marker and potential therapeutic target for Parkinson's disease," The Journals of Gerontology: Series A, vol. 75, no. 4, pp. 690-695, 2019.

[176] N. Rai, R. Kumar, G. R. Desai et al., "Relative alterations in blood-based levels of sestrin in Alzheimer's disease and mild cognitive impairment patients," Journal of Alzheimer's Disease, vol. 54, no. 3, pp. 1147-1155, 2016.

[177] W. Yi, Y. Zhang, Y. Guo, D. Li, and X. Li, "Elevation of Sestrin-2 expression attenuates sevoflurane induced neurotoxicity," Metabolic Brain Disease, vol. 30, no. 5, pp. 11611166, 2015.

[178] C. Zhang, W. Sun, J. Li et al., "Loss of sestrin 2 potentiates the early onset of age-related sensory cell degeneration in the cochlea," Neuroscience, vol. 361, pp. 179-191, 2017.

[179] Y.-S. Chen, S. D. Chen, C. L. Wu, S. S. Huang, and D. I. Yang, "Induction of sestrin2 as an endogenous protective mechanism against amyloid beta-peptide neurotoxicity in primary cortical culture," Experimental Neurology, vol. 253, pp. 6371, 2014.

[180] E. Ebnoether, A. Ramseier, M. Cortada, D. Bodmer, and S. Levano-Huaman, "Sesn2 gene ablation enhances susceptibility to gentamicin-induced hair cell death via modulation of AMPK/mTOR signaling," Cell Death Discovery, vol. 3, no. 1, pp. 1-9, 2017.

[181] J. Hanus, H. Zhang, D. H. Chen et al., "Gossypol acetic acid prevents oxidative stress-induced retinal pigment epithelial necrosis by regulating the FoxO3/Sestrin2 pathway," Molecular and Cellular Biology, vol. 35, no. 11, pp. 1952-1963, 2015.

[182] Y. Fan, Y. Xing, L. Xiong, and J. Wang, "Sestrin2 overexpression alleviates hydrogen peroxide-induced apoptosis and oxidative stress in retinal ganglion cells by enhancing Nrf2 activation via Keap1 downregulation," Chemico-Biological Interactions, vol. 324, p. 109086, 2020.

[183] B. U. Peres, A. J. H. Allen, A. Shah et al., "Obstructive sleep apnea and circulating biomarkers of oxidative stress: a cross-sectional study," Antioxidants, vol. 9, no. 6, p. 476, 2020.

[184] S. Cofta, H. M. Winiarska, A. Płóciniczak et al., "Oxidative stress markers and severity of obstructive sleep apnea," in Pulmonology, pp. 27-35, Springer, 2019.

[185] R. Jiang, Q. Wang, H. Zhai, X. du, S. Sun, and H. Wang, "Explorating the involvement of plasma Sestrin2 in obstructive sleep apnea," Canadian Respiratory Journal, vol. 2019, 6 pages, 2019.

[186] L. Bai, C. Sun, H. Zhai et al., "Investigation of urinary Sestrin2 in patients with obstructive sleep apnea," Lung, vol. 197, no. 2, pp. 123-129, 2019.

[187] J. Chai, J. Wang, R. Jiang et al., "Diagnostic value of Sestrin2 in patients with obstructive sleep apnea," Metabolic Syndrome and Related Disorders, vol. 18, no. 8, pp. 362-367, 2020.

[188] R. Bhattacharjee, L. Kheirandish-Gozal, G. Pillar, and D. Gozal, "Cardiovascular complications of obstructive sleep apnea syndrome: evidence from children," Progress in Cardiovascular Diseases, vol. 51, no. 5, pp. 416-433, 2009.

[189] P. J. Barnes, "Oxidative stress-based therapeutics in COPD," Redox Biology, vol. 33, article 101544, 2020.
[190] V. Mishra, J. Banga, and P. Silveyra, "Oxidative stress and cellular pathways of asthma and inflammation: therapeutic strategies and pharmacological targets," Pharmacology \& Therapeutics, vol. 181, pp. 169-182, 2018.

[191] D.-W. Zhang, Y. Y. Wei, S. Ji, and G. H. Fei, "Correlation between sestrin2 expression and airway remodeling in COPD," BMC Pulmonary Medicine, vol. 20, no. 1, pp. 1-10, 2020.

[192] Y. Kang, C. Chen, X. Hu et al., "Sestrin2 is involved in asthma: a case-control study," Allergy, Asthma \& Clinical Immunology, vol. 15, no. 1, pp. 1-6, 2019.

[193] Z. Tsilogianni, J. R. Baker, A. Papaporfyriou et al., "Sirtuin 1: endocan and sestrin 2 in different biological samples in patients with asthma. Does severity make the difference?," Journal of Clinical Medicine, vol. 9, no. 2, p. 473, 2020.

[194] Y. Yang, S. Cuevas, I. Armando, and P. Jose, “Azithromycin induces sestrin2 expression through Nrf2 signaling pathway in lung epithelial cells stimulated with cigarette smoke extract (869.11)," The FASEB Journal, vol. 28, no. S1, p. 869.11, 2014.

[195] X. Jiang, X. Liu, Y. Liu, F. U. Hui, X. Liu, and Z. Yang, "Protective effect and mechanism of Sestrin2 in the development of pulmonary alveolar type II epithelial cell injury induced by cigarette smoking," Chinese Journal of Comparative Medicine, vol. 28, no. 5, pp. 46-52, 2018.

[196] N. Olson, M. Hristova, N. H. Heintz, K. M. Lounsbury, and A. van der Vliet, "Activation of hypoxia-inducible factor-1 protects airway epithelium against oxidant-induced barrier dysfunction," American Journal of Physiology-Lung Cellular and Molecular Physiology, vol. 301, no. 6, pp. L993-L1002, 2011.

[197] J. Heidler, A. Fysikopoulos, F. Wempe et al., "Sestrin-2, a repressor of PDGFR $\beta$ signalling, promotes cigarette-smokeinduced pulmonary emphysema in mice and is upregulated in individuals with COPD," Disease Models \& Mechanisms, vol. 6, no. 6, pp. 1378-1387, 2013.

[198] F. Wempe, S. de-Zolt, K. Koli et al., "Inactivation of sestrin 2 induces TGF- $\beta$ signaling and partially rescues pulmonary emphysema in a mouse model of COPD," Disease Models \& Mechanisms, vol. 3, no. 3-4, pp. 246-253, 2010.

[199] A. Tomasovic, N. Kurrle, D. Sürün et al., "Sestrin 2 Protein Regulates Platelet-derived Growth Factor Receptor $\beta$ (Pdgfr $\beta$ ) Expression by Modulating Proteasomal and Nrf2 Transcription Factor Functions*," Journal of Biological Chemistry, vol. 290, no. 15, pp. 9738-9752, 2015.

[200] W. D. Tan, H.-M. Shen, and W. F. Wong, "Dysregulated autophagy in COPD: a pathogenic process to be deciphered," Pharmacological Research, vol. 144, pp. 1-7, 2019.

[201] A. L. B. C. Morsch, E. Wisniewski, T. F. Luciano et al., "Cigarette smoke exposure induces ROS-mediated autophagy by regulating sestrin, AMPK, and mTOR level in mice," Redox Report, vol. 24, no. 1, pp. 27-33, 2019.

[202] J. S. Lee, S. J. Park, Y. S. Cho, J. W. Huh, Y. M. Oh, and S. D. Lee, "Role of AMP-activated protein kinase (AMPK) in smokinginduced lung inflammation and emphysema," Tuberculosis and Respiratory Diseases, vol. 78, no. 1, pp. 8-17, 2015.

[203] W. Cui, Z. Zhang, P. Zhang et al., "Nrf2 attenuates inflammatory response in COPD/emphysema: crosstalk with Wnt3a/ $\beta$-catenin and AMPK pathways," Journal of Cellular and Molecular Medicine, vol. 22, no. 7, pp. 3514-3525, 2018.

[204] T. Iizuka, Y. Ishii, K. Itoh et al., "Nrf2-deficient mice are highly susceptible to cigarette smoke-induced emphysema," Genes to Cells, vol. 10, no. 12, pp. 1113-1125, 2005. 
[205] X.-p. Tong, Y.-x. Ma, D.-n. Quan, L. Zhang, M. Yan, and X.r. Fan, "Rosemary extracts upregulate Nrf2, Sestrin2, and MRP2 protein level in human hepatoma HepG2 cells," Evidence-Based Complementary and Alternative Medicine, vol. 2017, 7 pages, 2017.

[206] K.-B. Chen, Y. Xuan, W. J. Shi, F. Chi, R. Xing, and Y. C. Zeng, "Sestrin2 expression is a favorable prognostic factor in patients with non-small cell lung cancer," American Journal of Translational Research, vol. 8, no. 4, pp. 1903-1909, 2016.

[207] J. Ye, M. Wang, Y. Xu et al., "Sestrins increase in patients with coronary artery disease and associate with the severity of coronary stenosis," Clinica Chimica Acta, vol. 472, pp. 51-57, 2017.

[208] I. Tsilioni, A. S. Filippidis, T. Kerenidi, A. V. Budanov, S. G. Zarogiannis, and K. I. Gourgoulianis, "Sestrin-2 is significantly increased in malignant pleural effusions due to lung cancer and is potentially secreted by pleural mesothelial cells," Clinical Biochemistry, vol. 49, no. 9, pp. 726-728, 2016. 